University of Tennessee Health Science Center

UTHSC Digital Commons

\title{
Vestibular Evoked Myogenic Potentials and Postural Control in Adults with Age-Related Hearing Loss
}

Steven Michael Doettl

University of Tennessee Health Science Center

Follow this and additional works at: https://dc.uthsc.edu/dissertations

Part of the Speech and Hearing Science Commons, and the Speech Pathology and Audiology Commons

\section{Recommended Citation}

Doettl, Steven Michael (http://orcid.org/0000-0002-2291-1660), "Vestibular Evoked Myogenic Potentials and Postural Control in Adults with Age-Related Hearing Loss" (2020). Theses and Dissertations (ETD). Paper 517. http://dx.doi.org/10.21007/etd.cghs.2020.0504.

This Dissertation is brought to you for free and open access by the College of Graduate Health Sciences at UTHSC Digital Commons. It has been accepted for inclusion in Theses and Dissertations (ETD) by an authorized administrator of UTHSC Digital Commons. For more information, please contact jwelch30@uthsc.edu. 


\title{
Vestibular Evoked Myogenic Potentials and Postural Control in Adults with Age- Related Hearing Loss
}

\begin{abstract}
It is well-documented that auditory thresholds decrease with age, which can be referred to as Age-related hearing loss (ARHL). ARHL is one of the most common conditions affecting older adults and is associated with several conditions including decreased postural stability and falls. Age-related changes to auditory function have been attributed to, through histopathological study, specific degradation of the sensory, supporting, and afferent structures of the cochlea. Vestibular function, as measured through vestibular evoked myogenic potentials (VEMPs) also show decreases with age. VEMPs are a measurement of evoked potentials generated by auditory stimulation of the utricle and saccule measured through a reduction in muscle potentials. Similar to ARHL, age-related changes to the vestibular sensory and supporting structures with specific degeneration of the saccule, utricle, otoconia, primary vestibular afferents have also been noted. Significant decreasesin postural control with age are also welldocumented in literature attributed to multifactorial changes in function. Previous studies have found associations between vestibular dysfunction and hearing loss in specific pathologies. Additional associations between ARHL and decreased postural control have also been documented. However, only limited data has been reported evaluating the association of $A R H L$, decreased vestibular function, and postural control.

The results of this study indicated significant differences in VEMP findings for individuals with ARHL compared to an age-matched group with normal hearing. Additionally, significant correlations were noted across groups with decreases in hearing thresholds associated with decreases in VEMP amplitude and prolongation of VEMP latency. No significant differences between the ARHL and normal hearing group were noted for postural control measures. No significant correlations were noted for hearing thresholds and postural measures. These results are consistent with previous literature describing concomitant vestibular dysfunction in ARHL and other types of SNHL. The long-term goal of this line of this study is to evaluate the use of auditory function as a predictor of increased risk of falls and a possible criterion for subsequent balance function testing and intervention as needed as a means to reduce the risk and occurrence of falls in people with ARHL.
\end{abstract}

Document Type

Dissertation

Degree Name

Doctor of Philosophy (PhD)

Program

Speech and Hearing Science

Research Advisor

Patrick N. Plyler, Ph.D.

\section{Keywords}

Hearing Loss, Postural Control, Vestibular

\section{Subject Categories}

Communication Sciences and Disorders | Medicine and Health Sciences | Speech and Hearing Science | Speech Pathology and Audiology

This dissertation is available at UTHSC Digital Commons: https://dc.uthsc.edu/dissertations/517 
University of Tennessee HeAlth Science Center

DOCTORAL DisSERTATION

\section{Vestibular Evoked Myogenic Potentials and Postural Control in Adults with Age-Related Hearing Loss}

Author:

Steven Michael Doettl
Advisor:

Patrick N. Plyler, Ph.D.

A Dissertation Presented for The Graduate Studies Council of

The University of Tennessee Health Science Center

in Partial Fulfillment of Requirements for the Doctor of Philosophy degree from

The University of Tennessee

in

Speech and Hearing Science: Hearing Science

College of Graduate Health Sciences

May 2020 
Copyright (C) 2020 by Steven Michael Doettl.

All rights reserved.

Modified with permission Masters/Doctoral Thesis LaTeX Template

Version $2.5(8 / 27 / 2017)$

http://www.LaTeXTemplates.com Creative Commons License CC BY-NC-SA 3.0 


\section{Acknowledgements}

I want to thank my mentor, Dr. Patrick Plyler. I am grateful for his guidance throughout this experience. Dr. Devin McCaslin was also instrumental in the development of my career in research and I am in debt for his contributions. I would also like to thank the rest of my committee, Dr. Ashley Harkrider, Dr. Patti Johnstone, and Dr. Mark Hedrick for their continued support, mentorship, and guidance. I also would like to thank my colleagues for their support throughout the program and on a daily basis. Finally, I will of course thank my family for their support and love as always and during my work on this degree. 


\title{
Abstract
}

\author{
Steven Michael Doettl \\ Vestibular Evoked Myogenic Potentials and Postural Control in \\ Adults with Age-Related Hearing Loss
}

It is well-documented that auditory thresholds decrease with age, which can be referred to as Age-related hearing loss (ARHL). ARHL is one of the most common conditions affecting older adults and is associated with several conditions including decreased postural stability and falls. Age-related changes to auditory function have been attributed to, through histopathological study, specific degradation of the sensory, supporting, and afferent structures of the cochlea. Vestibular function, as measured through vestibular evoked myogenic potentials (VEMPs) also show decreases with age. VEMPs are a measurement of evoked potentials generated by auditory stimulation of the utricle and saccule measured through a reduction in muscle potentials. Similar to ARHL, age-related changes to the vestibular sensory and supporting structures with specific degeneration of the saccule, utricle, otoconia, primary vestibular afferents have also been noted. Significant decreases in postural control with age are also well-documented in literature attributed to multifactorial changes in function. Previous studies have found associations between vestibular dysfunction and hearing loss in specific pathologies. Additional associations between ARHL and decreased postural control have also been documented. However, only limited data has been reported evaluating the association of ARHL, decreased vestibular function, and postural control.

The results of this study indicated significant differences in VEMP findings for individuals with ARHL compared to an age-matched group with normal hearing. Additionally, significant correlations were noted across groups with decreases in hearing thresholds associated with decreases in VEMP amplitude and prolongation of VEMP latency. No significant differences between the ARHL and normal hearing group were noted for postural control measures. No significant correlations were noted for hearing thresholds and postural measures. These results are consistent with previous literature describing concomitant vestibular dysfunction in ARHL and other types of SNHL. The long-term goal of this line of this study is to evaluate the use of auditory function as a predictor of increased risk of falls and a possible criterion for subsequent balance function testing and intervention as needed as a means to reduce the risk and occurrence of falls in people with ARHL. 


\section{Contents}

1 INTRODUCTION 1

1.1 Auditory, Vestibular, and Postural Control Measurement

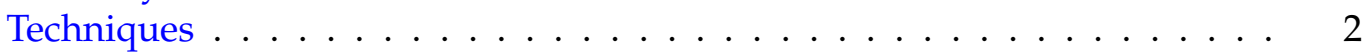

1.2 Histopathological Changes in the Cochlea and Vestibule with Aging . . . . 3

1.3 Auditory and Vestibular Function Changes with Aging . . . . . . . . . . 4

1.4 Postural Control Changes with Aging . . . . . . . . . . . . . . 4

1.5 Concomitant Dysfunction of the Auditory, Vestibular, and/or Postural Con-

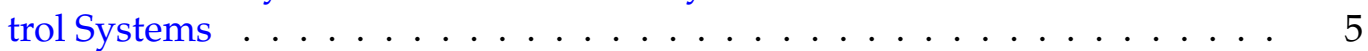

1.6 Rationale for This Study . . . . . . . . . . . . . . 5

2 REVIEW OF LITERATURE $\quad 7$

2.1 Histopathological Changes in the Cochlea and Vestibule with Aging . . . 7

2.2 Auditory Function Changes with Aging . . . . . . . . . . . . . . . . . . 10

2.3 Vestibular Function Changes with Aging . . . . . . . . . . . . . . . . . 11

2.4 Postural Control Changes with Aging . . . . . . . . . . . . . . . 13

2.5 Concomitant Dysfunction of the Auditory, Vestibular, and/or Postural Control Systems in Noise-Induced Hearing Loss . . . . . . . . . . . . . . . 14

2.6 Concomitant Dysfunction of the Auditory, Vestibular, and/or Postural Control Systems in Sensorineural Hearing Loss . . . . . . . . . . . . . 17

2.7 Concomitant Dysfunction of the Auditory, Vestibular, and/or Postural Control Systems in Age-Related Hearing Loss . . . . . . . . . . . . . . . 18

3 THEORETICAL FRAMEWORK

4 METHODOLOGY $\quad 24$

4.1 Participants . . . . . . . . . . . . . . . . . . . . 24

4.2 Audiologic Assessment . . . . . . . . . . . . . . . . . . 25

4.3 Vestibular Assessment . . . . . . . . . . . . . . . . . 25

4.4 Postural Control Assessment . . . . . . . . . . . . . . . . 26

5 RESULTS 28

6 DISCUSSION AND CONCLUSIONS $\quad 38$

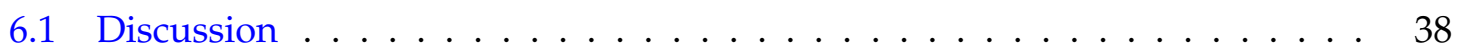

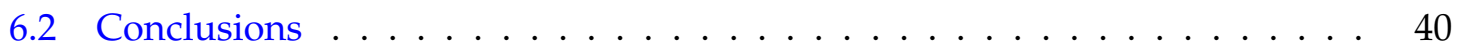


List of References

Vita 


\section{List of Tables}

5.1 Independent Samples T-Test for Group Differences in Mean on VEMP Measures. . . . . . . . . . . . . . . . . . . . . . . . . 29

5.2 Independent Samples T-Test for Group Differences in Mean for Postural Stability Measures . . . . . . . . . . . . . . . . . . . . 32

5.3 Pearson Correlation for Mid-Frequency PTA and VEMP Measures . . . . . . 32

5.4 Benjamin-Hochberg Correction for Multiple Comparisons for VEMP Mea-

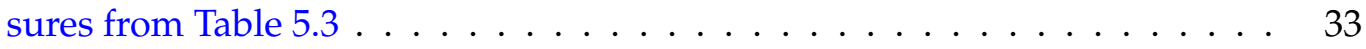

5.5 Pearson Correlation for ARHL Group for Low-Frequency PTA, High- Frequency PTA and Hearing Loss Slope and VEMP amplitudes . . . . . . . . . 33

5.6 Benjamin-Hochberg Correction for Multiple Comparisons for Low-Frequency PTA from Table $5.5 \ldots \ldots \ldots$. . . . . . . . . . . . . . . . . . 33

5.7 Benjamin-Hochberg Correction for Multiple Comparisons for High-Frequency PTA from Table $5.5 \ldots \ldots \ldots \ldots \ldots$. . . . . . . . . . . . . . . . . 34

5.8 Benjamin-Hochberg Correction for Multiple Comparisons for Hearing Loss Slope from Table $5.5 \ldots \ldots \ldots \ldots \ldots \ldots \ldots$ 


\section{List of Figures}

5.1 Mean cVEMP Amplitude for Group. . . . . . . . . . . . . . . . . . . . . . 30

5.2 Mean cVEMP Amplitude of Normal and ARHL Groups . . . . . . . . . . . . 31

5.3 Correlation of Mid-Frequency PTA to cVEMP P1/N1 Amplitude . . . . . . 34

5.4 Correlation of Mid-Frequency PTA to oVEMP P1 Latency . . . . . . . . . . . 35

5.5 Correlation of cVEMP P1/N1 Amplitude and HL Slope for the ARHL Group

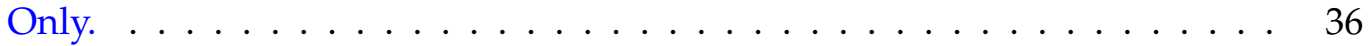

5.6 Correlation of oVEMP N1/P1 Amplitude and HL Slope for the ARHL Group

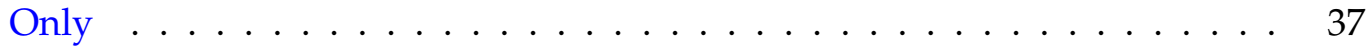




\section{List of Abbreviations}

$\begin{array}{ll}\text { ABR } & \text { Auditory brainstem response } \\ \text { ANOVA } & \text { Analysis of variance } \\ \text { ARHL } & \text { Age-related hearing loss } \\ \text { ASHA } & \text { American-Speech-Language Hearing Association } \\ \text { CDC } & \text { Centers for Disease Control and Prevention } \\ \text { cVEMP } & \text { Cervical vestibular evoked myogenic potentials } \\ \text { dB } & \text { Decibel } \\ \text { EP } & \text { Endocochlear potential } \\ \text { EMG } & \text { Electromyography } \\ \text { ENG } & \text { Electronystagmography } \\ \text { FRT } & \text { Functional Reach Test } \\ \text { HF-SNHL } & \text { High-frequency sensorineural hearing loss } \\ \text { HL } & \text { Hearing loss } \\ \text { HLS } & \text { Hearing loss slope } \\ \text { htDVAT } & \text { Head thrust dynamic visual acuity test } \\ \text { Hz } & \text { Hertz } \\ \text { ISSHL } & \text { Idiopathic sudden sensorineural hearing loss } \\ \text { KD } & \text { King-Devich } \\ \text { k } \Omega & \text { Kiloohm } \\ \text { msec } & \text { Millisecond } \\ \text { NIDCD } & \text { National Institute on Deafness and Other Communication Disorders } \\ \text { NIHL } & \text { Noise induced hearing loss } \\ \text { oVEMP } & \text { Ocular vestibular evoked myogenic potentials } \\ \text { PTA } & \text { Pure tone average } \\ \text { RT } & \text { Romberg testing } \\ \text { SCC } & \text { Semicircular canal } \\ \text { SCM } & \text { Sternocleidomastoid } \\ \text { SNHL } & \text { Sensorineural hearing loss } \\ \text { SPL } & \text { Sound pressure level } \\ \text { uV } & \text { Microvolt } \\ \text { VCR } & \text { Vestibulo-collic reflex } \\ \text { VEMP } & \text { Vestibular evoked myogenic potential } \\ \text { VOR } & \text { Vestibulo-ocular reflex } \\ \text { VSR } & \text { Vestibulo-spinal reflex } \\ \text { WHO } & \text { World Health Organization } \\ & \end{array}$




\section{Chapter 1}

\section{INTRODUCTION}

According to the National Institute on Deafness and Other Communication Disorders (NIDCD), presbycusis or age-related hearing loss (ARHL) is the one of the most common conditions affecting older adults. Approximately 1 in 3 people in the United States between 65-and 74-years of age have hearing loss (Age-Related Hearing Loss 2015, NIDCD). In addition to disruption in communication ability, ARHL has also been associated with many other conditions such as cognitive decline and depression (Lin, Yaffe, et al., 2013; Mener et al., 2013). Recently, there has been a suggestion that ARHL may also be associated with decreased postural control and an increased risk of falls (Lin and Ferrucci, 2012; Viljanen et al., 2009). The Centers for Disease Control (CDC) reports that falls are the leading cause of fatal and nonfatal injuries among adults aged 65 years and older with 27,000 older adults dying because of falls and 2.8 million treated in emergency departments for fall-related injuries with approximately 800,000 resulting in hospitalization (Bergen, Stevens, and Burns, 2016).

Histopathological studies describe specific changes to the sensory and supporting structures of the auditory portion of the cochlea with age and associated decreased auditory function (Schuknecht, 1964; Schuknecht and Gacek, 1993; Bai et al., 1997; Lin and Ferrucci, 2012). Vestibular structures have also been noted to degrade with age with histopathological studies describing changes to the vestibular sensory and supporting structures with specific degeneration of the saccule, utricle, otoconia, and primary vestibular afferents with increasing age (Schuknecht, Icarashi, and Gacek, 1965; Johnsson, 1971). Vestibular function tests, such as vestibular evoked myogenic potentials (VEMPs) also exhibit significant age-effects (Su et al., 2004; Basta, Todt, and Ernst, 2007; Nguyen, Welgampola, and Carey, 2010; Li et al., 2015). Postural control decreases with age have been extensively described in the literature and attributed to several different reported multifactorial changes in the musculoskeletal, neuromuscular, and sensory systems (Horak, Shupert, and Mirka, 1989; Maki, Holliday, and Fernie, 1990; Hageman, Leibowitz, and Blanke, 1995; Steffen, Hacker, and Mollinger, 2002; Kear, Guck, and McGaha, 2017).

Currently, there is ample evidence to support histopathologic changes in auditory 
and vestibular structures, changes in auditory and vestibular function, and reduced postural control with age; however, the relationship between auditory and vestibular degradation with age and reduced postural control, if any exists, has not been fully explored. Based on the previous studies describing these changes with age and histopathological evidence, it could be postulated that degradation of auditory structures is concomitant with degradation of vestibular structures. Additionally, while decreased postural control and increased fall risk with age are likely multifactorial, concomitant vestibular degradation may be a significant factor in people with ARHL. Previous literature has suggested a relationship between auditory and vestibular function exists for specific pathologies such as auditory neuropathy/dys-synchrony, sudden idiopathic hearing loss, ototoxicity, and noise-induced hearing loss through both histopathological and vestibular function studies (Wang and Young, 2007; Hong et al., 2008; Sazgar et al., 2006; Kumar, Vivarthini, and Bhat, 2010; Tseng and Young, 2012).

\subsection{Auditory, Vestibular, and Postural Control Measurement Techniques}

Auditory function is measured clinically through behavioral evaluation using audiometry. The American Speech-Hearing-Language Association (ASHA) Guidelines for Manual Pure-Tone Threshold Audiometry describe the procedure as manual air-conduction measurements at 250, 500, 1000, 2000, 3000, 4000, 6000, and $8000 \mathrm{~Hz}(125 \mathrm{~Hz}$ under some circumstances) plus bone-conduction measurements at octave intervals from $250 \mathrm{~Hz}$ to 4000 $\mathrm{Hz}$ and at $3000 \mathrm{~Hz}$ as needed (Guidelines for Manual Pure-Tone Threshold Audiometry 2020, ASHA). An audiometric threshold, or the softest sound a person can respond to at least $50 \%$ of the time, is determined at each frequency indicated. Additional analysis of audiometric thresholds includes audiometric pure tone averages (PTAs). PTAs consistent of an average on multiple thresholds at varying frequencies which can provide an assessment of degree of hearing loss across frequency and is often used to correlate audiometric results as well or to qualify hearing loss for research purposes and statistical analysis.

Vestibular function is measured by a variety of testing equipment and procedures including electronystagmography, videonystagmography, and rotary chair testing, auditory brainstem response, electrocochleography, Video Head Impulse Testing, and VEMPs. VEMP testing has been identified as pertinent specifically when evaluating for concomitant auditory and vestibular dysfunction (Wang and Young, 2007; Hong et al., 2008; Sazgar et al., 2006; Kumar, Vivarthini, and Bhat, 2010; Tseng and Young, 2012). VEMP evaluations (cervical and ocular) provide an evaluation of saccule and the inferior division of the vestibular nerve of the VIII cranial nerve and utricle and superior division of the vestibular nerve of the VIII cranial nerve function using the electrophysiologic evaluation of the vestibulo-spinal and vestibulo-ocular reflexes, respectively (Colebatch and Halmagyi, 1992; Colebatch, Halmagyi, and Skuse, 1994; Todd et al., 2007; Curthoys, Vulovic, and Manzari, 2012). VEMPs are elicited through auditory stimulation using high intensity 
stimuli and measured through surface electrodes and provide a measurement of evoked potentials originating in the utricle and saccule and are the only available method for assessing the otolithic organs in a quantitative manner. A relationship between auditory thresholds and VEMP results can be postulated to exist based on theories describing a bi-modal process with degradation of both the auditory and vestibular structures in the cochlea related to shared phylogenetic age, anatomical proximity, and/or similar physical stimulation processes.

Balance function is predicated on integration of sensory input from the visual, vestibular, and proprioceptive systems and motor control of both reflexive and voluntary muscle movements. Specific vestibular reflexes are integral in maintaining balance consisting of the vestibulo-ocular reflex (VOR), vestibulo-collic reflex (VCR), and the vestibulospinal reflex (VSR). Postural control can be measured through many different qualitative and quantitative measures. For example, a classic postural control assessment is the Romberg Test (RT), which evaluates postural control of positioning, requiring healthy functioning dorsal columns of the spinal cord as well as normal vestibular function. The RT can been can be used to evaluate for loss of vestibulo-spinal reflex and gross axial motor control and has been validated for use with normative values and age-related changes (Thyssen et al., 1982; Røgind et al., 2003). The Functional Reach Test (FRT) is another measure of postural control that has been identified as a pertinent and efficient measure of postural control with age-reference normative and cutoff values for intervention noted (Duncan, Weiner, et al., 1990; Duncan, Studenski, et al., 1992; Mann et al., 1996). The FRT has also been validated for use in the identification of peripheral vestibular dysfunction (Mann et al., 1996).

\subsection{Histopathological Changes in the Cochlea and Vestibule with Aging}

Age-related hearing loss has been outlined through histopathological study of animal and human temporal bones by Schuknecht, 1964 to include four distinct subtypes related to the underlying mechanisms investigated. The subtypes consisted of sensory, neural, metabolic, and cochlear conductive presbycusis. These categories were then expanded by Schuknecht and Gacek, 1993 to include mixed and indeterminate types. The classification of the subtypes, while not significantly useful for the clinical diagnosis and treatment of presbycusis, do highlight the important underlying pathophysiology of presbycusis. That is, that there is a variety of degenerative changes to the auditory system that can and will result in decreased hearing as we age. Ohlemiller, 2004 described this further by revisiting Schuknecht's subtypes in light of the current state of the literature regarding presbycusis and postulated that, for most individuals, mixed and/or indeterminate presbycusis is suspected and is the result of a myriad of factors throughout their life including but not limited to noise exposure, overall health status, genetic predisposition, and illnesses. 
While the auditory effects of presbycusis have been well-described, the possible association with vestibular dysfunction has not received as much attention. Schuknecht, Icarashi, and Gacek, 1965 first described specific degeneration of the saccule in subjects with known presbycusis. Additionally, Johnsson, 1971 described the degenerative histopathological changes that occur in the vestibular system with age postulating that vestibular degeneration may be an integral part of presbycusis. Park et al., 2001 also investigated age-related changes in vestibular neural ganglion with results suggesting degeneration of the neural ganglia associated with age.

\subsection{Auditory and Vestibular Function Changes with Aging}

It is well-documented that auditory function degrades over time as a function of presbycusis and many other factors such as overall health status, noise exposure, and specific otologic and non-otologic pathologies. Previous studies have indicated increasing prevalence of hearing loss with an increase in age with upwards of 45

Vestibular function has also been well-documented to decline with age with VEMP testing as a measure. Previous studies have indicated a significant age effect, especially at 50-years of age and older with cVEMP testing specifically for P1/N1 amplitude and N1 latency across the age span of adulthood (Su et al., 2004; Basta, Todt, and Ernst, 2007; Nguyen, Welgampola, and Carey, 2010; Li et al., 2015). oVEMP testing also has been shown to exhibit age effects with significant difference noted for N1/P1 amplitude and N1 latencies also specifically at 50-years of age (Nguyen, Welgampola, and Carey, 2010; Li et al., 2015). Interestingly, Nguyen, Welgampola, and Carey, 2010 also reported amplitude results as a continuous variable for age and found no significant associations, suggesting that age in isolation may not be the overwhelming factor in vestibular degradation over time. Additional factors such as gender and race have also been suggested as significant factors; however, only minimal data supports those suppositions at this time (Li et al., 2015).

\subsection{Postural Control Changes with Aging}

Reduced postural control has been well-described with aging related to a myriad of underlying pathologies and/or degrading systems. Horak, Shupert, and Mirka, 1989 describe a generalized aging effect associated with neural degeneration, peripheral vestibular loss, and other components. Additional studies across several age groups indicate both spontaneous and induced-sway measures demonstrated significant decreases with age and significant difference between groups with decreased measures of postural control (Thyssen et al., 1982; Maki, Holliday, and Fernie, 1990; Hageman, Leibowitz, and Blanke, 1995). Specific clinical postural control measures such as the Six-minute Walk Test, Berg Balance Scale, Timed Up and Go Test, comfortable- and fast-speed walking, and the Functional Reach Test (FRT) also demonstrated significant decreases in postural stability with age 
with good sensitivity for identifying the phenomenon (Duncan, Weiner, et al., 1990; Duncan, Studenski, et al., 1992; Steffen, Hacker, and Mollinger, 2002; Bischoff et al., 2003; Hageman, Leibowitz, and Blanke, 1995; Kear, Guck, and McGaha, 2017). Romberg testing (RT) has also been identified as a sensitive measure of aged-related decreases in postural control (Thyssen et al., 1982; Røgind et al., 2003). The Functional Reach Test specifically, has been extensively studied in the literature with results suggesting significant age effects as well published age-related normative data (Duncan, Weiner, et al., 1990; Duncan, Studenski, et al., 1992; Hageman, Leibowitz, and Blanke, 1995).

\subsection{Concomitant Dysfunction of the Auditory, Vestibular, and/or Postural Control Systems}

Several studies have found associations between hearing loss and vestibular function for specific pathologies such as noise induced hearing loss and sudden idiopathic sensorineural hearing loss and for profound sensorineural hearing loss (Hong et al., 2008; Wang and Young, 2007; Kumar, Vivarthini, and Bhat, 2010; Tseng and Young, 2012; Xu et al., 2016). However, very few studies have investigated ARHL. Zuniga et al., 2012, evaluated older adults with ARHL using both cervical and ocular VEMPs. Zuniga and colleagues found that while cervical VEMP amplitudes correlated with SNHL, they found no significant relationship to ocular VEMP recorded using tap-evoked stimulation. While it has been previously suggested that anatomical and physiologic differences make the utricle not as susceptible, if at all, to the same degenerative affects as the saccule, conflicting data has been reported.

Previous studies have also suggested a significant association between postural control and ARHL. Viljanen et al., 2009 indicated that increased hearing loss correlated with a higher risk for falls and decreased postural control in older twins with no indication of significant association with genetic factors. Lin and Ferrucci, 2012 also reported a significant association between hearing loss and the odds of reported falls with every 10-dB increase in hearing loss increasing the odds a fall. These two studies suggest a significant relationship between decreased hearing with age and decreased postural stability, that which could be postulated as an associative relationship.

\subsection{Rationale for This Study}

Both auditory and vestibular structures degrade with age. Age is also a factor in both decreased hearing and decreased vestibular function. Hearing loss has been associated with decreased postural control and increased risk of falls in older adults. Previous studies have found associations between vestibular function and hearing loss in specific pathologies, however there is a paucity of data regarding vestibular function and ARHL. Current evidence suggests that vestibular function, specifically saccular function, may decrease as 
a function of degradation of hearing, but data is limited. Additional study is needed to further evaluate vestibular function and auditory function in ARHL as well the relationship, if any exists, to reduced postural control.

Specifically, this study is designed to further evaluate auditory function, vestibular function, postural control in adults with ARHL, using audiometric testing, cVEMPs, oVEMPs, and postural control measures. The central hypothesis is that adults with ARHL will exhibit reduced saccule and utricle function as measured by VEMPs and reduced postural control as measured by postural control measures and the results will be correlated with the audiometric thresholds. This association is postulated to be due to concomitant histopathological changes in the auditory and vestibular structures associated with ARHL and resulting in reduced sensory information for maintaining postural control. The results of this study will provide specific information about the association between the degree of ARHL with saccule and utricle function as measured by cVEMPs and oVEMPs and postural control measures. The long-term goal of this study is to improve the understanding of the relationship between ARHL and reduced postural control and improve identification criteria and management for adults with hearing loss at risk for reduced vestibular function and postural control and increased falls risk.

The specific research questions for this study are as follows:

1. Is there a significant difference in saccule/utricle function as measured by cVEMP and oVEMP testing and postural control between older adults with normal hearing and older adults with ARHL?

2. Is there a significant correlation between audiometric thresholds and saccule/utricle function as measured by cVEMP and oVEMP and/or postural control as measured by RT and FRT? 


\section{Chapter 2}

\section{REVIEW OF LITERATURE}

\subsection{Histopathological Changes in the Cochlea and Vestibule with Aging}

It has been well-documented that cellular changes occur in the auditory structures of the cochlea as part of the aging process. These pathological changes in the cochlea from aging were first described by Crowe, Guild, and Polvogt, 1934 and Saxen, 1937 for which they evaluated human cadaver temporal bones and attributed age-related hearing loss (presbycusis) to degradation of the organ of Corti and/or of the spiral nerve ganglion, specifically at the basal turn of the cochlea. These changes were further described and expanded by Schuknecht, 1964 to include four distinct subtypes of presbycusis related to the underlying pathology; sensory, neural, metabolic, and cochlear conductive. Sensory presbycusis is described as atrophy of the organ of Corti and spiral ganglion at the basal turn of the cochlea. Specifically, a decline of the number and condition of the hair cells and supporting cells of the cochlea. Additionally, the loss of hair cells progresses to a point that which this degradation continues to also include loss of spiral ganglion cells as well. Neural presbycusis is described as loss of the neuronal population in the central nervous system as described across a lifespan. Genetic factors may play a role in neural presbycusis as well as the time of onset and degree of degradation appear to very greatly across the population. Neural presbycusis is defined by quantifying the number of first order neurons present. Metabolic presbycusis describe defects in the physical and chemical processes of the cochlea, specifically the function of stria vascularis, which is responsible at least in part, for endolymphatic potentials and oxidative enzyme production through bioelectric and biochemical reactions, respectively. These metabolic changes result in a reduction of the endocochlear potential (EP) and thus cochlear function. Mechanical (cochlear conductive) presbycusis represents a degeneration of the mechanics of the cochlear motion, possibly a narrowing or stiffening of the basilar membrane over time resulting in reduced stimulation of auditory hair cells. Further expansion of these pathological changes and descriptions were added again by Schuknecht and Gacek, 1993 to include mixed and indeterminate types.

Ohlemiller, 2004 revisited Schuknecht's subtypes considering the current state of 
the literature regarding presbycusis at that time. He concluded that while even with advancements in our ability to evaluate cell structure that Schuknecht's descriptions are still relevant in the investigation of related pathophysiology of age-related hearing loss. These classifications of the subtypes of presbycusis and other theories involving the underlying pathology in presbycusis do highlight the importance of understanding the underlying cochlear pathophysiology. That is, that there is a variety of degenerative changes to the cochlea that can and will result in decreased cochlear function with age. Mixed and indeterminate types of presbycusis represent either a combination of the above noted types or an inability to qualify into a specific type or types, respectively. Using these classic foundational studies from Schuknecht and colleagues and the updated descriptions and techniques described by Ohlemiller, 2004, it could be postulated that, for most individuals, mixed or indeterminate presbycusis is suspected and is the result of a myriad of factors throughout their life including normal cellular aging, genetic predisposition, environmental factors, and overall health status.

Schuknecht, Icarashi, and Gacek, 1965 were the first to describe physiologic changes in the saccule as a result of aging. Through histopathologic post-humus evaluation of the temporal bones of an aged cat, aged dog, and a human that suffered from progressive ARHL, Schuknecht and colleagues identified specific degeneration of the saccule related to aging. In the aged cat, a loss of roughly half of the vestibular hair cells and supporting cells of the macula sacculi were noted bilaterally and symmetrically. In the aged dog, similar bilateral and symmetric degeneration was noted with loss of half of the vestibular hair cells and damaged supporting cells; however, additional degeneration of the sensory epithelium of saccule with displacement and atrophy of the otolithic membrane was also noted. In the aged man, similar bilateral and symmetric degeneration was noted, again with some important distinctions. Significant collapse of the saccular wall with adherence to the macule along with severe macula degeneration accompanied by approximately $50 \%$ vestibular hair cell loss was noted.

Overall Schuknecht, Icarashi, and Gacek, 1965 summarized the possible changes as loss of macula sacculi, atrophy of the otolithic membrane, loss of vestibular hair cells, flattened sensory epithlium, and saccular wall collapse. Interestingly, they also noted that utricle and semicircular canals (SCCs) appeared to be intact. This was theorized to be due to the phylogenetic development, for which the organ of corti and the saccule develop together to form the pars inferior later in development than the pars superior associated with the SCCs and the utricle. That is that phylogenetically younger physiologic structures are more susceptible to degeneration than older structures, which is thus represented with similar cellular degeneration of the organ of corti and saccule with age. In this case, then the utricle and the SCCs make up the pars superior, and thus are not as susceptible to degenerative changes. At the time of the study it was postulated that these cellular changes would be either metabolic, cytochemical, enzymatic, or microanatomical in nature.

Johnsson, 1971 further described the degenerative histopathological changes that 
occur in the vestibular system with age. He examined the temporal bones of 150 participants from the fetal ages to 97-years of age at autopsy. Twenty-four samples were from participants with a history of presbycusis. Of these samples, he noted an "obvious degeneration of the saccular nerve network, while degeneration of the utricle was only mild". Additional degenerative changes in the saccule were described as loss of otoconia. Johnsson, 1971 described almost invariable loss of otoconia in samples of participants 60-years of age or older. Samples 30-years of age or younger rarely demonstrated any loss of otoconia. Samples 30-60-years of age showed varying degrees of otoconia degeneration. Utricular otoconia had comparable but much less effect noted; however, utricular otoconia was difficult to assess in this study due to anatomical differences and the specimen preparation. Johnsson, 1971 further elaborated that the loss of otoconia is possibly due to "mechanical wear" and could be associated with increase rates of dizziness seen in older adults. Additionally, it was proposed that the saccule, due to its vertical alignment may be more prone to loss of otoconia than the utricle and its horizontal configuration due to gravitational effects.

Park et al., 2001 investigated age-related changes in vestibular neural ganglion with age. Using 20 sectioned temporal bones from samples ranging in age from 2-88-years of age, a documented decrease in vestibular neurons from an average of 28,000 cells in youth to roughly 23,000 cells above 60 -years of age was noted. These results were also consistent with the findings from Johnsson, 1971 concerning the age range of decline beginning after 30-years of age and especially after 60-years of age. Loss of macula sacculi, atrophy of the otolithic membrane, loss of vestibular hair cells, flattened sensory epithelium, and saccular wall collapse were all noted; however, utricular and SCC structures appeared to be intact. Based on these findings it was suggested that presbycusis results in degeneration of vestibular sensory structures (specifically the saccule), as well as, auditory sensory structures. It was suggested that this pattern of degeneration was likely due to the phylogenetic development, for which the organ and corti and the saccule develop together to form the pars inferior. Whereas the utricle and the SCCs make up the pars superior, thus are not as susceptible to degenerative changes.

Additional theories outside of Schuknecht's subtypes also exist and center around a few key elements of age-related cochlear changes. Mitochondrial degeneration and resulting associative oxidative stress, release of free-radicals, and subsequent apoptosis has been suggested as a key factor in age-related cellular changes in the cochlea (Someya and Prolla, 2010). Fetoni et al., 2011 also provided a comprehensive review of the possible underlying pathogenesis of presbycusis using animal models including reduced vascularization of the stria vascularis, oxidative stress, apoptosis, damage to collagen, immune pathology, noise, gonadal hormones, and other dysfunctions. Specific studies such as Bai et al., 1997 evaluated 34 temporal bones from 17 participants with hearing and 17 participants without hearing loss noting that 14 of 17 samples with hearing loss showed a mitochondrial deletion compared to only 7 of 17 showing a deletion in the normal hearing group. The authors suggested that the presence of a specific mitochondrial deletion $(4,977 \mathrm{bp})$ is significantly associated with aging and at least some forms of presbycusis. 


\subsection{Auditory Function Changes with Aging}

Brant and Fozard, 1990 evaluated audiometric thresholds from 813 adult males from 20-95years of age, broken into 7 age groups for age related changes in audiometric results. They evaluated each subject from $0.125-8 \mathrm{kHz}$ over a 20 -year period with serial audiograms. The results indicated changes in audiometric thresholds in all age groups evaluated with a range of $5.1 \mathrm{~dB}(0.5 \mathrm{~Hz})$ to $21.1 \mathrm{~dB}(8 \mathrm{kHz})$ of change for 20 -year-olds and up to $22.9 \mathrm{~dB}$ $(0.5 \mathrm{~Hz})$ to $84.5 \mathrm{~dB}(8 \mathrm{kHz})$ for 80 -year-olds. These results indicated that the high frequencies were more susceptible to decrease, especially with increasing age. Overall this study indicates that there is a significant decline in auditory function with aging that can be measured accurately and reliability through changes in pure tone audiometric thresholds and the process begins in the third decade of life, is progressive, and is predominant in the highest frequencies.

Epidemiological studies also describe ARHL in the adult population. Cruickshanks et al., 1998 expanded the literature by completing an epidemiological study for the prevalence of ARHL across gender. The participants consisted of 3753 adults 48-92-years of age. The evaluation consisted of otoscopy, tympanometry, pure-tone air- and bone-conduction audiometry in a sound treated booth, and a questionnaire of hearing health related topics such as medical history, noise exposure, other risk factors, and perceived handicap. The results indicated that the prevalence of hearing loss was $45.9 \%$ with an increase in odds of hearing loss with age. There was also a gender difference noted with men having much higher odds of having hearing loss than women, even when adjusted for age, education, noise exposure and occupation.

Helzner et al., 2005 also evaluated a large subject population (muli-site) to evaluate for prevalence and risk factors associated with hearing loss. His group evaluated across gender, like Cruickshanks et al., 1998, but also included race as a factor. They completed audiometric evaluation of 2052 subjects 73-84-years of age, including 660 Caucasian men, 631 Caucasian women, 310 African American men, and 451 African American women from a Health ABC study and metropolitan areas of Pittsburg, Pennsylvania and Memphis, Tennessee. Pure-tone threshold testing was completed with hearing loss qualified as greater than $25 \mathrm{~dB} H \mathrm{HL}$ average hearing loss at 500,1000, and $2000 \mathrm{~Hz}$ and greater than $40 \mathrm{~dB}$ HL average hearing loss at 2000, 4000, and $8000 \mathrm{~Hz}$. Additional demographic information was also collected including medical history, noise exposure, medication usage, and lifestyle factors. In agreement with Cruickshanks et al., 1998, the results indicated ARHL is quite prevalent in older adults with $59.9 \%$ indicated hearing loss by the defined criteria for just the 500,1000, and $2000 \mathrm{~Hz}$ average and 76.9\% using only the 2000, 4000, and $8000 \mathrm{~Hz}$ average. Caucasian men had the highest prevalence of hearing loss with Caucasian women, African American men, and African American women following in that order. Gender difference were consistent with the previous findings from Cruickshanks et al., 1998. Other factors were also suggested as significant in ARHL including diabetes mellitus, cerebrovascular disease, smoking, poorer cognitive status, occupational noise exposure, and ear surgery. 
Agrawal, Platz, and Niparko, 2008 completed a national cross-sectional survey of hearing loss across the United State with adults 20-69-years of age. Like Helzner et al., 2005 hearing loss was qualified as greater than $25 \mathrm{~dB} H \mathrm{HL}$ average hearing loss in the low- to midfrequency range $(500,1000,2000$, and $4000 \mathrm{~Hz})$ and high-frequency range $(3000,4000$, and $6000 \mathrm{~Hz}$ ). Even with this younger participant population, $16.1 \%$ of participants across the age span have hearing loss in the low- to mid-frequency range with $43 \%$ (males) and $20 \%$ (females) in the 60-69-year age group. Again, males had a higher prevalence of hearing loss than women, and Caucasians had a higher prevalence of hearing loss than African Americans with additional factors such as smoking, noise exposure, and cardiovascular risks (hypertension) associated. Additionally, a follow-up study by Gopinath et al., 2009 aimed to confirm these associated factors. Their evaluation of 3654 participants 49 -years of age or older included initial evaluations and follow-up at 5- and 10-year intervals including face-to-face interviews to obtain comprehensive medical history and additional demographic information. Pure-tone audiometry determined audiometric hearing thresholds at 500, 1000, 2000, and $4000 \mathrm{~Hz}$ defining hearing loss as an average greater than 25 $\mathrm{dB} H \mathrm{HL}$ and moderate to severe hearing loss as a greater than $40 \mathrm{~dB} \mathrm{HL}$ in the better ear. Results from this study were consistent with Agrawal, Platz, and Niparko, 2008 suggested that hearing loss is quite prevalent in the adult population, increasing with age, and associated with several additional factors.

\subsection{Vestibular Function Changes with Aging}

Su et al., 2004 investigated the effect of aging on VEMP results. Eighty normal participants 7-75-years of age with no history of hearing or vestibular problems. Participants were then divided into 4 groups: Group I - <20-years of age, Group II 21-40-years of age, Group III 41-60- years of age, and Group IV - >60-years of age. cVEMP testing was completed using an air-conducted click stimulus at 95dBnHL in a supine position with head lifts used for sternocleidomastoid (SCM) stimulation. Electromyography (EMG) levels were monitored to maintain a level of 50-200uV. Latencies, peak amplitude and interaural amplitude differences were recorded. The results for the first 40 ears in each group were analyzed for percentage of present cVEMP responses. Groups I and II presented with $98 \%$ present cVEMP responses. Group II had $90 \%$ present responses. Group IV has $60 \%$ present responses with a significant difference noted between the other three groups. Additionally, of the present responses in each group the latency and amplitude data was analyzed. Results indicated a significant aging effect for P1/N1 amplitude and N1 latency.

Basta, Todt, and Ernst, 2007 aimed to investigate gender and age on cVEMP amplitude. They evaluated 64 normal participants $20-76$ years of age (38 females and 26 males) dividing each participant into 3 age groups (Group I: 20-40-years of age; Group II: 41-60years of age; Group III: 60-76-years of age). cVEMPs were recorded ipsilateral with airand bone-conducted stimuli. EMG of the SCM was recorded ipsilateral. The participants were instructed to turn the head to the contralateral shoulder and hold the position to maintain stable SCM activation throughout recording. EMG recordings were then used to 
normalize the cVEMP P1/N1 amplitude. The authors reported a 100\% cVEMP response rate (air- and/or bone-conducted stimuli) across participants. Results of this study were consistent with previous studies with significant differences in cVEMP P1/N1 amplitude between the each of the 3 age groups with amplitude values decreasing with increasing age. No significant difference was noted in P1/N1 amplitude with stimulation technique or across gender.

Janky and Shepard, 2009 also evaluated the effect of aging on cVEMP parameters, specifically threshold measures. Forty-six participants 20-76-years of age were separated into five age groups. cVEMP responses were obtained at both maximal stimulus levels for $500 \mathrm{~Hz}$ tone-burst and at threshold to various stimuli (click, 250, 500, 750, and 1000 $\mathrm{Hz}$ tone-burst). cVEMP testing was completed with the participant in a seated with headturns for SCM activation using a blood pressure cuff as a guide to maintain flexion. No significant differences were noted for age for N1 latency or P1/N1 amplitude. However, a significant difference was noted for P1 latency and threshold for various stimulation frequencies. Age was also significantly positively correlated with the cVEMP threshold and negatively correlated with P1/N1 amplitude. The authors also noted that the cVEMP response rate also decreased with increased age.

Nguyen, Welgampola, and Carey, 2010 evaluated age effects in both cVEMPs and oVEMPs. Fifty-three normal participants (24 males and 29 females) 20-70-years of age were divided into three age groups (20-30- years of age; $31-50$-years of age; and $>50$-years of age). Air-conducted stimuli (click and $500 \mathrm{~Hz}$ tone-burst) and vibration stimuli were used (midline at the hairline, $30 \%$ of the distance between the inion and nasion) for cVEMP and oVEMP testing respectively. cVEMP testing was completed with each participant semi recumbent (30-degree) and instructed to lift their heads up from rest for SCM flexion. EMG was recorded and included for amplitude rectification. oVEMPs were completed with the participant semi recumbent (30-degree) and instructed to look upward with maximal gaze for inferior oblique flexion. The authors reported that participants older than 50years of age had significantly decreased cVEMP P1/N1 and oVEMP N1/P1 amplitudes. Interestingly, this group also evaluated age and VEMP amplitudes as continuous variables with no significant associations indicated. No significant age effects were noted for cVEMP or oVEMP latencies or asymmetry ratios.

Li et al., 2015 evaluated age-related changes in both oVEMPs and cVEMPs as well as with other related health factors. 314 community dwelling participants 26-92-years of age were evaluated with 250 completing cVEMP testing and 257 completing oVEMP testing. Extensive demographic information was also collected regarding gender, race, cardiovascular risk factors (hypertension, diabetes, hyperlipidemia), and smoking history. cVEMPs were completed using an air-conducted $500 \mathrm{~Hz}$ tone-burst stimuli in a 30-degree supine position, with head lifts to activate the SCMs and EMG rectification. oVEMPs were completed using tap-evoked stimuli (head-taps delivered manually via reflex hammer at Fz) in a 30-degree supine position with a 20-degree upward gaze. The results were analyzed for percentage of present responses. Latency, peak amplitude, and asymmetry ratio values 
were also recorded. Significant differences were noted for cVEMP corrected P1/N1 amplitude across age categories indicating decreased amplitude with increasing age. oVEMP results indicated a significant difference across age categories for both N1/P1 amplitude and N1 latency values with a decrease in amplitude and prolongation of N1 latency with increasing age. Additional significant effects for gender for cVEMPs and race for oVEMPs were noted. All other values and demographic information were not significant.

\subsection{Postural Control Changes with Aging}

It has been well-described that overall balance function declines with age due to multifactorial changes in the musculoskeletal, neuromuscular, and sensory systems. Horak, Shupert, and Mirka, 1989 describe the components of decreased postural control in the elderly population. The authors reviewed the state of the literature at the time of publication and described a generalized aging effect on balance mechanisms, but specifically highlighted neural degeneration and peripheral vestibular loss as well other components that contribute to decreased postural control with age. Peripheral vestibular loss specifically has been reported and summarized by the authors as reduced number of vestibular hair cells, neural ganglion cells, and eighth nerve fibers which are commonly associated with aging as well as the possible presence of pathologic peripheral vestibular lesions across a lifespan. It was also noted that the degree of peripheral vestibular loss is not always specifically related to reduced balance function overall due to compensatory mechanisms and the redundant nature of postural control across sensory systems (vestibular, visual, proprioception).

Maki, Holliday, and Fernie, 1990 specifically evaluated age-related decreases in postural sway. They evaluated 64 subjects consisting of normal healthy young participants, normal healthy elderly participants, and elderly participants with a history of falling for spontaneous postural sway and induced postural sway with applied postural perturbations. The results indicated both the spontaneous and induced-sway measures demonstrated significant decreases with age. Some additional specific relationships in the elderly participants were also noted including significant difference between fallers and non-fallers in the spontaneous sway condition, which suggests the fallers may have a more severe disruption of the postural control mechanisms with a theoretical critical point of becoming a faller. One of the more well-known postural control measures used in both clinical and research forums is Romberg testing (RT). Thyssen et al., 1982 evaluated 110 healthy individuals ages 20-69-years of age and reported excellent test-retest reliability as well age-related decreases in sway across the age span. Røgind et al., 2003 also evaluated the use of RT in 1952 healthy individuals 20-70-years of age and reported significant increase in sway with age across gender.

Steffen, Hacker, and Mollinger, 2002 evaluated the elderly population for several well-known clinical test protocols for postural control. The authors recruited 96 normal healthy community dwelling participants 61-89-years of age and completed the Six-minute 
Walk Test, Berg Balance Scale, Timed Up and Go Test, and Comfortable- and Fast-Speed Walking. The results indicated no significant gender effects, but did suggest that age is a significant factor in the results for all the tests evaluated. The authors also noted that of the postural tests evaluated, the Six-Minute Walk test, The Timed Up and Go Test, and the Fast Speed Walking measurements showed high test-retest reliability. Hageman, Leibowitz, and Blanke, 1995 also evaluated postural control and aging as well and any gender effect through postural control measures. The authors evaluated 48 normal adults in two groups. The groups consisted of younger adults (20-35-years of age) and older adults (60-75-years of age) balanced with equal male and female participants. The subjects were evaluated using five force platform measures (movement time, path length to targets, and sway area under eyes open, eyes closed, and visual referenced conditions) and the Functional Reach Test (FRT) in centimeters. The results were not significant for gender, but did indicate a significant difference between the groups with decreased measures in all six measures of postural control. Duncan, Weiner, et al., 1990 and Duncan, Studenski, et al., 1992 also reported specifically on the use of the FRT and reported a strong association with decreased FRT scores and recurrent falls in the elderly population.

\subsection{Concomitant Dysfunction of the Auditory, Vestibular, and/or Postural Control Systems in Noise-Induced Hearing Loss}

Noise-induced hearing loss (NIHL) has been found to correlate significantly with histopathological changes in vestibular structures, reduced postural stability and vestibular dysfunction noted with ENG, VNG, and VEMP testing. Histopathological study has indicated specific cochlear destruction from noise exposure. Animal model studies have linked specific histopathological changes in the vestibular structures following noise exposure. Akdogan et al., 2009 evaluated vestibular effects of noise exposure using animal models and histopathological evaluation of temporal bones. 10 adult albino guinea pigs were exposed to either intermittent or continuous noise. Following exposures, the temporal bones were evaluated with characteristic changes in the macula, degeneration of epithelial cells, and cell apoptosis were noted with a significant increase in these changes noted for the continuously exposed group. However, there is discrepancy within the literature regarding these findings. Specifically, McGill and H. F. Schuknecht, 1976 evaluated the temporal bones for 14 human ears with NIHL. Their results indicated degenerative changes at the basal turn of the cochlear duct with damage to hair cells, supporting cells and inner and outer sulcus cells in all cases correlating with auditory function. However, no specific pattern of significant vestibular degeneration within cochlea was noted as least suggesting the possibility that NIHL and vestibular dysfunction were no connected at least on a histopathological level in these cases.

Further evaluation of the relationship between NIHL and vestibular function in humans involved more specific peripheral vestibular function testing. Golz et al., 2001 examined 258 male military personnel with an average of 27-years of age with documented 
exposure to significant to both impact and impulse noise and 35 normal healthy controls. The NIHL participants were evaluated using audiometry (separated into groups of symmetric and asymmetric hearing loss) and electronystagmography (ENG). Significant differences in the presentation of vestibular symptoms (vertigo, dizziness, imbalance, etc.) and ENG findings were noted between the symmetric and asymmetric NIHL groups as well as the asymmetric NIHL group and the control group. Spontaneous nystagmus, positional nystagmus, reduced slow phase velocity, and caloric asymmetries were also noted in the NIHL groups. Increased ENG abnormalities also correlated with reported vestibular symptoms in the NIHL groups. Overall, the study suggested that exposure to both military impulse and impact noise poses a significant risk to vestibular function in addition to and concomitant with auditory function.

Wang and Young, 2007 evaluated vestibular dysfunction related to hearing loss looking specifically at chronic NIHL. Audiometry, stapedial, caloric, oculomotor, positional, and cVEMP testing was completed for twenty participants with NIHL ranging from 33-61-years of age with a range of 10-30-years of occupational noise exposure. NIHL was classified using diagnostic criteria from the College of Occupational and Environmental Medicine, with emphasis on the classic "notching" of the audiogram at 3000, 4000, or $6000 \mathrm{~Hz}$ with recovery at $8000 \mathrm{~Hz}$. Caloric testing was also completed for both cool and warm irrigations in both ears for each subject. Participants were then categorized into two groups based on the 4-frequency threshold (Group I - 26-40dB and Group II - 41-75dB). cVEMP testing was completed using an air-conducted $500 \mathrm{~Hz}$ tone-burst stimuli at $95 \mathrm{~dB}$ HL in a supine position, with head lifts to activate the SCMs. EMG levels were monitored and relayed to the subject to maintain and average of greater than 50uV EMG during recording. cVEMP results were evaluated for presence/absence. Results indicated a significant correlation between absent cVEMP responses and the audiometric threshold at $4000 \mathrm{~Hz}$. Overall cVEMP responses were absent with greater hearing loss suggesting that saccule dysfunction is a correlate of NIHL. Additionally, caloric test results were also abnormal in $45 \%$ of the subjects; however, no correlation with the $4000 \mathrm{~Hz}$ notch was noted. This suggests additional vestibular dysfunction outside of the saccule, which conflicts with other studies that have suggested vestibular dysfunction associated with hearing loss is likely restricted to the saccule.

Kumar, Vivarthini, and Bhat, 2010 also evaluated NIHL and cVEMP testing. A total of 30 participants with NIHL ranging from 30-40-years of age and 30 normal controls were evaluated. Audiometry, tympanometry, and acoustic reflex testing was completed on each subject. cVEMP testing was completed using an air-conducted click stimulus at 99dBnHL in an upright position with head turns used for SCM stimulation. EMG levels were monitored and relayed to the subject to maintain and average of $50-100 \mathrm{uV}$ EMG during recording. cVEMP results were evaluated for presence or absence and latency and amplitude. cVEMP was absent in $29 \%$ of the ears for the NIHL group with significant differences noted in both P1 and N1 latency (delayed) and P1/N1 amplitude (smaller) compared to the control group. Additionally, a significant correlation was noted between PTA of hearing loss and both P1 and N1 latency and P1/N1 amplitude of the 
cVEMP responses. This study further elaborated on the vestibular effects, specifically the saccule-collic response, from NIHL suggesting significant changes in vestibular function with increased hearing loss, at least due to prolonged noise exposure.

Tseng and Young, 2012 were the first study to evaluate both saccule and utricle function using cVEMPs and oVEMPs in NIHL. Thirty participants with NIHL ranging from 22-64-years of age and 30 normal age and gender matched controls. NIHL was classified using diagnostic criteria from the College of Occupational and Environmental Medicine. Otoscopy, audiometry, and caloric testing was completed on each participant. Caloric testing was completed for both cool and warm irrigations in both ears for each participant. cVEMP testing was completed using a bone-conducted $500 \mathrm{~Hz}$ tone-burst stimuli beginning at 128dB FL (force-level) in a supine position with head lifts used for SCM stimulation. EMG levels were monitored and relayed to the participant to maintain and average of greater than $50 \mathrm{uV}$ EMG during recording. oVEMPs were completed using a bone-conducted $500 \mathrm{~Hz}$ tone-burst stimuli beginning at 128dB FL (force-level) in an upright sitting position with a 30-degree upward gaze. The results were analyzed for percentage of normal responses using latency, peak amplitude, and asymmetry ratio values. cVEMP was abnormal in 50\% of the ears for the NIHL group. oVEMP was abnormal in $33 \%$ of the ears for the NIHL group. Calorics were abnormal in $25 \%$ of the ears for the NIHL group. Significant differences were noted in both P1 and N1 latencies (delayed) and P1/N1 amplitude (smaller) compared to the control group. Additionally, a significant difference in percentage of abnormal test results for audiometry, cVEMP, oVEMP, and calorics between the NIHL and the control group was noted. Importantly these results suggest the pars superior (utricle) is effected by NIHL, albeit to lesser degree than the pars inferior (saccule), and can be measured through changes in the oVEMP response. This study also suggested and established a sequence of degenerative changes in NIHL as measured with diagnostic testing with audiometry, cVEMP, oVEMP, and calorics.

Additional studies have also linked NIHL with decreased postural control. For example, Juntunen et al., 1987 evaluated 60 participants with NIHL for body-sway measurements and compared them to 115 healthy normal controls. All NIHL participants were military personnel that had documented history of decreased audiometric thresholds and firearm noise exposure. Pure tone audiometry was completed along with force-plate platform postural sway evaluations. Postural stability was measured with eyes open and eye closed. Specific demographic information regarding the history of noise-exposure was also obtained. The results indicated significantly worse audiometric thresholds and increased postural sway for the NIHL group compared to the normal control group. Additionally, the NIHL participants with more hearing loss exhibited more postural sway than the NIHL participants with milder hearing impairments. Both auditory and vestibular degeneration were noted with noise exposure with the vestibular disruption suggested as a likely subclinical disturbance of the vestibular structures.

Additionally, Kilburn, Warshaw, and Hanscom, 1992 evaluated 78 iron workers 
compared to 128 normal controls (histology technicians). Audiometric testing was completed and postural stability was measured on an uncovered floor with the subject's feet together and arms folded and a visual target 6 feet away for eyes open and in an eyes closed condition. Sway was measured using head mounted microphones and a sound emitting stylus documenting head movement in relation to sound. The results documented both decreased hearing, increased postural sway, and increased sway speed compared to the normal controls suggesting noise exposure having a deleterious effect on both auditory and balance function. A significant correlation was also found between hearing thresholds and sway speed in the NIHL group only.

\subsection{Concomitant Dysfunction of the Auditory, Vestibular, and/or Postural Control Systems in Sensorineural Hearing Loss}

Peripheral vestibular loss and concomitant hearing loss have also been described in the literature regarding sudden idiopathic sensorineural hearing loss (ISSHL). Hong et al., 2008 evaluated vestibular function in patients with ISSHL. Hong and colleagues evaluated 52 patients with ISSHL without vertigo using cVEMP and audiometric threshold data. The results indicated a significant correlation with the degree of hearing loss present (greater than $90 \mathrm{~dB} \mathrm{HL}$ and less than 55dB HL over $1000 \mathrm{~Hz}$ ) and absent cVEMP response. The authors suggested that based on their findings a subclinical deterioration of the saccule function is noted in ISSHL, likely due to an underlying shared pathophysiology, proximity, and/or shared function of the structures. They also noted a significant correlation with audiometric thresholds, specifically high-frequency hearing loss and saccular function as measured by cVEMP in ISSHL, suggesting a clear association between loss of both auditory and vestibular function during the disease process.

Specific study linking SNHL and vestibular function has also suggested a significant association. Sazgar et al., 2006 evaluated 50 adult participants (20-60-years of age) with high-frequency sensorineural hearing loss (HF-SNHL) and 18 normal healthy adult participants (20-60-years of age) as controls. Audiometric testing was completed using airand bone-conduction audiometry, speech discrimination, speech recognition thresholds, and tympanometry. Pure tone averages were then calculated for the $4000-8000 \mathrm{~Hz}$ results to indicate the HF-SNHL, then divided into three groups (Group I - 20-40dB, Group II - 41$60 \mathrm{~dB}$, and Group 3 - >61dB PTAs). cVEMP testing was completed using an air-conducted click stimulus at 110dBnHL in a supine position with head lifts and turns used for SCM stimulation. The results were analyzed for percentage of present cVEMP responses for the normal group, and each of the three groups of HL subjects. The normal group has $84.4 \%$ present responses while the HF-SNHL subjects demonstrated only $55.3 \%$ present cVEMP responses. Group I demonstrated $79.2 \%$ present responses with Group II at $38.7 \%$ and Group III at $52.4 \%$. Significant differences were noted between the normal controls and the overall HF-SNHL participants as well as for each of the three groups. Additionally, a significant difference was noted between Group I and both Group II and Group III. No 
significant difference was noted between Group II and Group III. These results were also evaluated for factors such as P1 and N1 latency and age with no major significant findings noted between the normal group and the HF-SNHL group or within the three HF-SNHL groups. Overall these results suggest an association between SNHL and saccular function, which may increase with decreased PTAs. Interestingly, in this study age was not a factor in cVEMP presence, which has been suggested in other literature. This is possibly due to the age range used in this study as opposed to considering older adults greater than 60-years of age with ARHL.

Xu et al., 2016 also looked specifically at SNHL and vestibular dysfunction. A retrospective analysis of 29 participants 18-63-years of age was completed. Each participant had been diagnosed with bilateral profound SNHL of >95dB HL PTA of 500, 1000, 2000, and $4000 \mathrm{~Hz}$ for each ear. Otoscopy and tympanometry were performed to rule out concomitant middle ear pathology prior to testing. cVEMP testing was completed using an air-conducted $500 \mathrm{~Hz}$ tone-burst stimuli beginning at $125 \mathrm{~dB}$ SPL ( $5 \mathrm{~dB}$ steps as needed to elicit a response) in a supine position, with head lifts to activate the SCMs. oVEMPs were completed using an air-conducted $500 \mathrm{~Hz}$ tone-burst stimuli beginning at $125 \mathrm{~dB}$ SPL (5dB steps as needed to elicit a response) in a supine position with an upward gaze. Latency and peak amplitude values were recorded. Caloric testing was also completed for both cool and warm irrigations in both ears for each participant. Forty healthy control ears were also evaluated for comparison. The results indicated a response rate for both cVEMP and oVEMP in the normal control group of $100 \%$. The response rate in the profound SNHL group was $44.4 \%$ for cVEMPs and $38.9 \%$ in oVEMPs, which was significantly different from the normal control group. Additionally, in subjects with profound SNHL, when VEMP responses were present, threshold, latency, and amplitude values were all noted to be abnormal. Specifically, cVEMP results indicated elevated thresholds, reduced amplitudes, and shortened latencies for both P1 and N1 compared to controls. oVEMP results indicated elevated thresholds and reduced N1/P1 amplitude compared to the controls.

\subsection{Concomitant Dysfunction of the Auditory, Vestibular, and/or Postural Control Systems in Age-Related Hearing Loss}

Zuniga et al., 2012 evaluated 51 older adults 70-years of age and older. Participants were categorized for age, gender, race, educational level, smoking status, hypertension, and noise exposure. Audiometric testing was completed from $500-8000 \mathrm{~Hz}$ at both ears and PTAs were calculated for mid-frequency $(500,1000,2000$, and $4000 \mathrm{~Hz})$, low-frequency $(250$ $-1000 \mathrm{~Hz})$, and high-frequency $(4000$ and $8000 \mathrm{~Hz})$ thresholds. Head Thrust Dynamic Visual Acuity testing (htDVAT) was completed for static and dynamic visual acuity. cVEMPs were completed using an air-conducted $500 \mathrm{~Hz}$ tone-burst stimuli at $125 \mathrm{~dB}$ SPL in a 30degree supine position, with head turns to activate the SCMs and rectified for electromyography (EMG) levels. oVEMPs were completed using tap-evoked (mini-taps with a reflex hammer) at 30-degree supine with 30-degree upward gaze. P1/N1 amplitudes were 
recorded. The results indicated a significant correlation of all 3 PTAs with cVEMP P1/N1 amplitudes, strongest for the high-frequency PTA. None of the PTAs were significantly correlated with oVEMP N1/P1 amplitude or htDVAT results. Significant correlations were also noted for age, smoking status, and noise exposure then corrected for each factor with only noise exposure remaining as a significant factor. Overall the results indicated a significant correlation between the degree of hearing loss and increased noise exposure with decreased saccular function. These results also suggested that utricular function as measured by oVEMPs may not be associated with either degree of hearing loss. However; it may be important to note that tap-evoked oVEMPs have been reported to have reduced test-retest reliability than acoustic evoked oVEMPs (Nguyen, Welgampola, and Carey, 2010).

Kurtaran et al., 2016 evaluated 63 adults (55-82-years of age, mean 65.1-years of age). The groups consisted of 31 participants with normal hearing with 32 participants with SNHL. Audiometric testing was completing 250-8000Hz. cVEMP testing was completed at 95dBHL with $500 \mathrm{~Hz}$ tone-burst stimuli, $4 \mathrm{msn}$ rise/fall time, and $2 \mathrm{msec}$ plateau with a repetition rate of 5.5 per second. A non-inverting electrode was placed on the upper third of the SCM and the inverting electrode was placed over the SCM tendon above the clavicle. The participants were positioned upright and instructed to turn their heads toward the contralateral side of the tested ear. SCM equalization was completed and EMG response of the muscle was recorded. Results indicated significant differences for P1 and N1 latencies and P1/N1 amplitude with the SNHL group exhibiting lower P1/N1 amplitude and increased P1 and N1 latencies compared to the normal hearing group.

In addition to studies suggesting a significant association between ARHL and vestibular dysfunction, a recent systematic review also evaluated the association between hearing loss and postural control in older adults. Agmon, Lavie, and Doumas, 2017 summarized that hearing loss resulted in slower gait speed, lower standing balance, lower walking endurance, more difficulties walking distances, and lower Short Physical Performance Battery scores. People with hearing loss also performed fewer chair stands, experienced greater difficulties in stair-climbing, and had lower physical activity and higher risk for falls. Additionally, the severity of the hearing loss was connected to a higher prevalence of difficulties in walking and falls decreased participation in the community, and a proclivity to ambulate in their closest environment.

Viljanen et al., 2009 investigated hearing loss as a predictor of falls and postural control in older female twins. Two-hundred and seventeen female twin pairs 63-76-years of age were evaluated using audiometric testing, falls over a 345-day period, and postural control measures. The results indicated that increased hearing loss (mean thresholds level for $500-4000 \mathrm{~Hz}$ in $\mathrm{dB}$ ) correlated with a higher risk for falls and decreased postural control. Further analysis of the twin factor indicated no significant association that would explain the correlation of hearing loss, falls, and postural control with genetic factors.

Lin and Ferrucci, 2012 also evaluated hearing and falls in the elderly population using a cross-sectional study of 2017 participants 40-69-years of age using audiometric testing and fall history. Audiometric testing was completed and a PTA of 500, 1000, 2000, 
and $4000 \mathrm{~Hz}$ was calculated with hearing loss determined by greater than $25 \mathrm{~dB}$ HL. Correction was made for other contributing factors such as demographic factors and postural control measures (Modified Romberg Test of Standing Balance on Firm and Compliant Surfaces). Using logistic regression, the unadjusted model indicated a significant association between hearing loss and the odds of reported falls with every 10-dB increase in hearing loss increasing the odds a fall. Even after adjustment for covariate factors the significance of the association did not substantially change. 


\section{Chapter 3}

\section{THEORETICAL FRAMEWORK}

Histopathological changes in the cochlea with aging results in decreased auditory function. The underlying theories proposed for this degeneration can be centered around Schuknecht's descriptions involving the types of presbycusis also known as ARHL. These theories, first proposed by Schuknecht, 1964 and further elaborated by Schuknecht and Gacek, 1993 and Ohlemiller, 2004 describe sensory, neural, metabolic, cochlear conductive, mixed and indeterminate types of presbycusis each with a different (or combination) underlying pathophysiology. Atrophy of the organ of Corti and spiral ganglion at the basal turn of the cochlea, decline of the number and condition of the hair cells and supporting cells of the cochlea, loss of spiral ganglion cells, loss of the neuronal population in the central nervous system, genetic factors, defects in the physical and chemical processes of the cochlea with reduction of the endocochlear potential, and a degeneration of the mechanics of the cochlear motion have all been associated with aging. Obviously the final two subtypes, mixed and indeterminate, represent either a combination of the above noted types or an inability to qualify into a specific type or types, respectively.

Additional theories outside of Schuknecht's subtypes describing mitochondrial degeneration and resulting associative oxidative stress, release of free-radicals, cell apoptosis, reduced vascularization of the stria vascularis, oxidative stress, damage to collagen, immune pathology, noise, and gonadal hormones also exist to explain the pathophysiology of ARHL (Someya and Prolla, 2010; Fetoni et al., 2011; Bai et al., 1997). Using these classic foundational studies from Schuknecht, 1964 and Schuknecht and Gacek, 1993 and the updated descriptions and techniques described Ohlemiller, 2004, Someya and Prolla, 2010, Fetoni et al., 2011, and Bai et al., 1997 it could be postulated that, for most individuals, mixed or indeterminate ARHL is suspected and is the result of a myriad of factors throughout their life including normal cellular aging, genetic predisposition, environmental factors, and overall health status.

While the underlying pathophysiology and auditory effects of ARHL have been well-described, the possibility of associated vestibular effects have only been minimally investigated. Schuknecht, Icarashi, and Gacek, 1965 were the first to describe physiologic changes in the vestibular with aging through histopathologic post-humus evaluation of the 
temporal bones. Overall, Schuknecht and colleagues described specific changes to the saccule in cochleas with noted presbycusis with the utricle and SCCs spared. Johnsson, 1971 agreed with these findings as he also described degenerative histopathological changes in the saccule beginning after 30-years of age and especially after 60-years of age that occur in the vestibular system with age most specifically to the saccule. Park et al., 2001 described additional degenerative changes to the vestibule with aging reporting a decrease in vestibular neurons from youth to above 60-years of age. Based on these studies, it could be postulated that presbycusis is a bi-modal process with degradation that will involve both the auditory and vestibular structures in similar fashion. This is based on a combination of factors involving age-related cellular changes, genetic predispositions, environmental factors, and overall health status as well as the loss of both sensory and supporting cells as well neural ganglia in both structures all resulting from the same underlying associated pathology.

As previously stated, reduced postural control is well-documented with age and is multifactorial in nature; however, identifying and managing possible vestibular changes with age as factor in reduced postural control could have over-reaching effects on fall risk and overall morbidity and mortality rates in the elderly population. To best accomplish this goal, further study is needed to quantify and qualify vestibular effects associated with ARHL using vestibular and postural control tests to further describe this phenomenon. Based on the aforementioned theoretical framework, the following hypotheses are stated:

1. Saccule function as measured by cVEMP amplitude will be significantly lower in the ARHL group than in the control group.

2. Utricle function as measured by oVEMP amplitude will be significantly lower in the ARHL group than in the control group.

3. Saccule function as measured by cVEMP latency will be significantly prolonged in the ARHL group than in the control group.

4. Utricle function as measured by oVEMP latency will be significantly prolonged in the ARHL group than in the control group.

5. Postural control as measured by KD Balance will be significantly worse in the ARHL group than in the control group.

6. Postural control as measured by Functional Reach will be significantly worse in the ARHL group than in the control group.

7. There will be a significant correlation between pure tone averages and saccule and utricle function as measured by cVEMP and oVEMP measures and/or postural control as measured by KD Balance and FRT.

The long-term goal of this study is to improve the understanding of ARHL and reduced postural control, specifically by evaluating vestibular function. By furthering this understanding, we aim to improve identification criteria and management for adults with 
ARHL who could be at increased risk for reduced postural control and falls specifically related to age-related vestibular dysfunction. The results of this study will provide specific information about the association between ARHL and saccule/utricle function as measured by VEMPs. The results of this study will also describe significant associations, if any exist, between the degree of hearing loss in ARHL with saccule/utricle function as measured by VEMPs and postural control measures. 


\section{Chapter 4}

\section{METHODOLOGY}

\subsection{Participants}

An a priori power analysis using G-Power 3.1.9.2 completed using independent samples $t$-tests design, an alpha level of 0.05 , and a large effect size $(f=0.80)$ showed that a total sample size of at least 42 (ears) is sufficient for a power of at least 0.80 (actual power $=0.8167878$ ). Additional a priori analysis using a one-tailed bivariate correlation design assuming individual dependent variables, an alpha level of 0.05 , and a large effect size (f $=0.40$ ) showed that a total sample size of at least 34 (ears) is sufficient for a power of at least 0.80 (actual power $=0.8011069$ ). In the absence of previous data, a large effect size (f $=0.40$ ) was chosen to maximize clinical relevance.

All participants were required to sign an informed consent form before participating in the study. Participation consisted of one study session lasting approximately 2 hours. Participants were from the Knoxville, TN area and were recruited via advertisements (flyers, social media) and phone recruitment from the UTHSC Audiology Clinic research study volunteer database. Group was determined through audiometric evaluation consisting of pure tone air- and bone-conduction threshold evaluation. Recruited participants consisted of 34 adults (50-70-years of age) with 16 males and 18 females. Two ears from 2 separate male participants were not included in the study due to significant asymmetry associated with previous diagnosed otologic pathology. Four additional participants exhibited asymmetry between ears and ABRs were completed to rule out retrocochlear pathology with all 4 exhibiting normal ABR results and included in the study.

For the purposes of this study each ear was considered separate for PTAs and VEMP values. Demographic information was collected regarding noise exposure (no significant noise exposure or recreational, military, and occupational noise exposure), education level (high school, undergraduate, graduate), diabetes, cardiovascular problems, treatment with ototoxic drugs, and chemotherapy. Participants with history of significant neurologic pathology, diabetes, or other conditions associated specifically with hearing loss and/or vestibular/balance dysfunction or noted specific congenital or pathological (Meniere's disease, labyrinthitis, otosclerosis, conductive or mixed hearing loss, history of sudden onset hearing loss, or documented noise-induced hearing loss) hearing loss and or history of 
significant specific balance or vestibular dysfunction (i.e. Parkinson's disease, vestibular neuritis, labyrinthitis, etc.) were excluded from the study. From the 34 recruited participants, 6 ears were removed from analysis due to either suspected pathology other than ARHL and/or statistical outlier status. Group 1 consisted of 33 ears with normal hearing. Group 2 consistent of 27 ears with hearing loss determined using the World Health Organization (WHO) definition a PTA of 500, 1000, 2000, and $4000 \mathrm{~Hz}$ of greater than $25 \mathrm{~dB}$ (Report of the Informal Working Group on Prevention of Deafness and Hearing Impairment Programme Planning, Geneva, 18-21 June 1991 2020).

\subsection{Audiologic Assessment}

Evaluation of the external ear canals was completed to ensure that each ear canal was free of cerumen or foreign material prior to testing. Immittance testing was completed using the Interacoustics Titan system (Interacoustics, Middlefart, Denmark). Tympanometry was completed to evaluate middle ear compliance and pressure to ensure participants did not have significant middle ear dysfunction. Acoustic reflex thresholds were obtained bilaterally to both ensure participants did not have significant conductive or retrocochlear pathology and/or confirm cochlear site of lesion. All audiometric testing was conducted in a sound-treated examination room (IAC; $2.2 \times 1.8$ meters) with pure-tone stimuli were delivered through ER-3A insert earphones with the appropriately sized ER3-14 disposable foam ear tips utilizing a GSI 61 audiometer (Grason-Stadler, Eden Prairie, MN). Thresholds ( $\mathrm{dB} \mathrm{HL}$ ) for air-conducted tones were recorded to determine the level of frequency-specific hearing sensitivity at octave and inter-octave intervals from $250 \mathrm{~Hz}$ to $8000 \mathrm{~Hz}$. Bone conduction testing was completed for $500-4000 \mathrm{~Hz}$ to rule out conductive pathology.

$\mathrm{ABR}$ testing was completed when indicated by significant audiometric asymmetry $(20 \mathrm{~dB}$ or greater asymmetry at 2 consecutive frequencies or $15 \mathrm{~dB}$ or greater asymmetry at 2 consecutive frequencies $2000-8000 \mathrm{~Hz}$, or unilateral tinnitus) using the ICS Charter EP 200 system (Natus, Inc, Pleasanton, CA) (Gimsing, 2010). Testing consisted of recording using a stimulus delivered through auditory means (insert earphone) with recording using surface electrodes placed on each mastoid and on the center forehead. The active electrode was mounted to the middle of the forehead (Fpz), the reference electrode to the ipsilateral mastoid (M1), and the ground to the contralateral one (M2). All electrode impedances were below $5 \mathrm{k} \Omega$. Clicks were $100 \mu \mathrm{s}$ in duration and presented in rarefaction polarity at $80 \mathrm{dBnHL}$ at a rate of $21.1 / \mathrm{s}$ and $77.7 / \mathrm{s}$ (rate-study).

\subsection{Vestibular Assessment}

oVEMPs provide an assessment of the VOR consisting of the sensory structures of the utricle, the primary afferent nerve (superior portion of the vestibular nerve branch of cranial nerve VIII), vestibular nuclei, oculomotor nuclei, and the inferior oblique muscle. cVEMPs provide an assessment of the VCR (and at least shared portions of the VSR arc) consisting 
of the sensory structures of the saccule, the primary afferent nerve (inferior branch of the vestibular nerve), vestibular nuclei, medial vestibular spinal tract, and the sternocleidomastoid muscle (SCM). VEMP responses.

cVEMPs were recorded using $500 \mathrm{~Hz}$ tone-burst stimuli at 95dBnHL both ears using the ICS Charter EP 200. $500 \mathrm{~Hz}$ was chosen at the stimulus frequency due to the highest reported response rate for the population being evaluated. cVEMPs were recorded using surface electrodes placed on the middle of the SCM, each sterno-clavical junction, and the forehead. An auditory signal of 95dBnHL utilizing a $500 \mathrm{~Hz}$ tone-burst was delivered to the ears via insert earphones. EMG monitoring (to maintain stable EMG) and amplitude was also measured using additional electrodes just below the active electrodes on the SCMs with EMG rectification/normalization employed. Recording consisted of 2100 sweep runs with the largest response marked to obtain values of latency (msec) for waves, P1, N1, and $\mathrm{P} 2$ and the amplitude values $(\mathrm{uV})$ for the P1/N1 complex.

oVEMP were recorded using surface electrodes placed at the belly of the inferior oblique tendons (inferior oblique muscles) on one side of each eye, just outside the epicanthi folds on the other side of each eye, and on the forehead. An auditory signal of 95dBnHL utilizing a $500 \mathrm{~Hz}$ tone-burst will be delivered to the ears via insert earphones. Recording consisted of 2 runs of 30 sweeps added to obtain values of latency (msec) for waves, N1 and $\mathrm{P} 1$ and the amplitude values (uV) for the N1/P1 complex.

\subsection{Postural Control Assessment}

The FRT has been identified as a pertinent and efficient measure of postural control with age-reference normative and cutoff values for intervention noted (Duncan, Weiner, et al., 1990; Duncan, Studenski, et al., 1992). FRT is a behavioral procedure designed to evaluate the status of postural control in a standing and reaching position. The FRT has also shown to be sensitive to vestibular dysfunction in the elderly population (Duncan, Studenski, et al., 1992). The FRT procedure consists of a yard stick attached to a wall at the participant's shoulder height. The participant was then positioned close to the wall to enable them to reach forward along the length of the yardstick with their feet together and their arm parallel to the floor. The participant was then instructed to make a fist and raise their arm to 90-degrees for an initial baseline measure. They were then instructed to reach as far forward as possible without taking a step or release their fist. The investigator would then note the distance in inches covered from the baseline position of the third metatarsal to furthest reach. The FRT score was then recorded as the distance in inches. This was completed with 3 trials and an average of the final 2 scores were recorded to allow for learning affect and test-retest differences.

RT was also completed as measure of postural sway utilizing the KD Balance Application (King-Devich Technologies, Inc., Downers Grove, IL) in concert with Apple IPod Touch using iOS 8 (Apple, Inc, Cupertino, CA). The KD Balance application is an FDA $510(\mathrm{~K})$ cleared mobile application used by athletic trainers, physical therapists, physicians, 
and other healthcare professionals to assess postural stability. The KD Balance application utilizes RT with a specific protocol consisting of double leg Romberg with eyes closed, single leg Romberg with left foot forward, and single leg Romberg with right leg forward. Each of the 3 conditions are completed with the IPod in a fastened chest harness. An objective balance score is automatically derived by detecting the individual's movements using the device's internal accelerometer. After instruction and a 5 second countdown, a 20 second recording with eyes closed for each conditions were completed. FRT and KD Balance Scores could not be obtained one 1 subject due to an acute lower back injury at the time of the visit. 


\section{Chapter 5}

\section{RESULTS}

cVEMP and oVEMP measures were averaged for the normal hearing and ARHL groups. For final analysis, group 1 consisted of 33 ears from participants age 50-70 with normal hearing with average age of 60.8 years (sd 6.287 years) and an average mid-frequency PTA of $14.8 \mathrm{~dB}$. Group 2 consisted of 27 ears from participants age 50-70 years with ARHL and an average age of 62.9 years (sd 4.984 years) with an average mid-frequency PTA of $39.9 \mathrm{~dB}$. All statistical analyses were completed using IBM SPSS Statistics 25. An independent samples t-test was completed preliminarily to evaluate for any significant difference in age between the groups. No significant difference between groups for age was noted ( $p$-value 0.153). The main study analysis was then completed using independent samples $\mathrm{t}$-tests to assess group mean differences for dependent variables. The independent variable was group with 2 levels (normal hearing, ARHL). The dependent variables were cVEMP P1/N1 Amplitude, cVEMP P1 Latency, cVEMP N1 Latency, cVEMP P2 Latency, oVEMP N1/P1 Amplitude, oVEMP N1 Latency, and oVEMP P1 Latency. Results indicated a significant group difference for cVEMP P1/N1 Amplitude and oVEMP N1/P1 amplitude with smaller amplitudes noted for the ARHL group with no significant difference was noted for group for all the other dependent variables (Table 5.1; Figures 5.1 and 5.2). The postural stability scores were averaged for the control and ARHL groups. The independent variable was group (combined left and right ears). Independent sample T-tests were then used to evaluate group mean differences for postural stability testing as measured by the dependent variables of KD Balance Double Leg score, KD Balance Tandem Right score, the KD Balance Tandem Left score, and the FRT score. The results indicated no significant group mean differences (Table 5.2).

Additional analyses were completed using Pearson correlation to evaluate the relationship of the mid-frequency PTA to the dependent variables. The results of the correlational analyses with Benjamin Hochberg correction for multiple comparisons indicated significant correlations for cVEMP amplitude and oVEMP P1 latency (Tables 5.3 and 5.4; Figures 5.3 and 5.4). Based on these results and results from previous studies additional follow-up analysis was also completed using Pearson correlation to further evaluate the configuration of hearing loss (low-frequency PTA, high-frequency PTA, and Hearing Loss Slope) to the dependent variables cVEMP P1/N1 Amplitude and oVEMP N1/P1 Amplitude. Low-frequency PTA was calculated using the threshold data for 250, 500, and 
Table 5.1: Independent Samples T-Test for Group Differences in Mean on VEMP Measures.

\begin{tabular}{lccccccc}
\hline $\begin{array}{l}\text { VEMP } \\
\text { Measures }\end{array}$ & $\mathbf{t}$ & df & $\begin{array}{c}\text { P- } \\
\text { Value }\end{array}$ & $\begin{array}{c}\text { Mean } \\
\text { Diff. }\end{array}$ & $\begin{array}{c}\text { Std. } \\
\text { Error } \\
\text { Diff. }\end{array}$ & $\begin{array}{c}\text { 95\% Confidence } \\
\text { Interval of the } \\
\text { Difference }\end{array}$ \\
\hline $\begin{array}{l}\text { cVEMP P1 } \\
\text { Latency }\end{array}$ & -0.900 & 58 & 0.372 & -0.246 & 0.273 & -0.791 & 0.300 \\
$\begin{array}{l}\text { LVwer } \\
\text { cVEMP N1 }\end{array}$ & 0.070 & 58 & 0.945 & 0.041 & 0.586 & -1.132 & 1.214 \\
$\begin{array}{l}\text { Latency } \\
\text { cVEMP P2 }\end{array}$ & -0.507 & 58 & 0.614 & -0.576 & 1.136 & -2.850 & 1.699 \\
$\begin{array}{l}\text { Latency } \\
\text { cVEMP }\end{array}$ & 2.725 & 58 & 0.008 & 44.994 & 16.514 & 11.938 & 78.051 \\
$\begin{array}{l}\text { Amplitude } \\
\text { oVEMP N1 }\end{array}$ & -0.938 & 57 & 0.352 & -0.235 & 0.251 & -0.738 & 0.267 \\
$\begin{array}{l}\text { Latency } \\
\text { oVEMP P1 }\end{array}$ & -1.279 & 57 & 0.206 & -0.462 & 0.362 & -1.187 & 0.262 \\
$\begin{array}{l}\text { Latency } \\
\text { oVEMP } \\
\text { Amplitude }\end{array}$ & 2.343 & 53.005 & 0.023 & 0.957 & 0.408 & 0.138 & 1.777 \\
\hline
\end{tabular}

$1000 \mathrm{~Hz}$. High-frequency PTA was calculated using 4000, 6000, and 8000Hz. Hearing Loss Slope (HLS) was calculated by subtracting the high-frequency average (3000, 4000 and $6000 \mathrm{~Hz}$ ) from the bilateral low-frequency average (250, 500 and $1000 \mathrm{~Hz})$ (Hornsby, Johnson, and Picou, 2011). The results of the correlational analyses (with Benjamin Hochberg correction for multiple comparisons) indicated a significant correlation for Low-frequency PTA with cVEMP P1/N1 amplitude only, High-frequency PTA with oVEMP N1/P1 amplitude only, however the HLS was significantly correlated with both the cVEMP P1/N1 amplitude and oVEMP N1/P1 amplitude (Tables 5.5, 5.6, 5.7, and 5.8; Figures 5.5 and 5.6). 


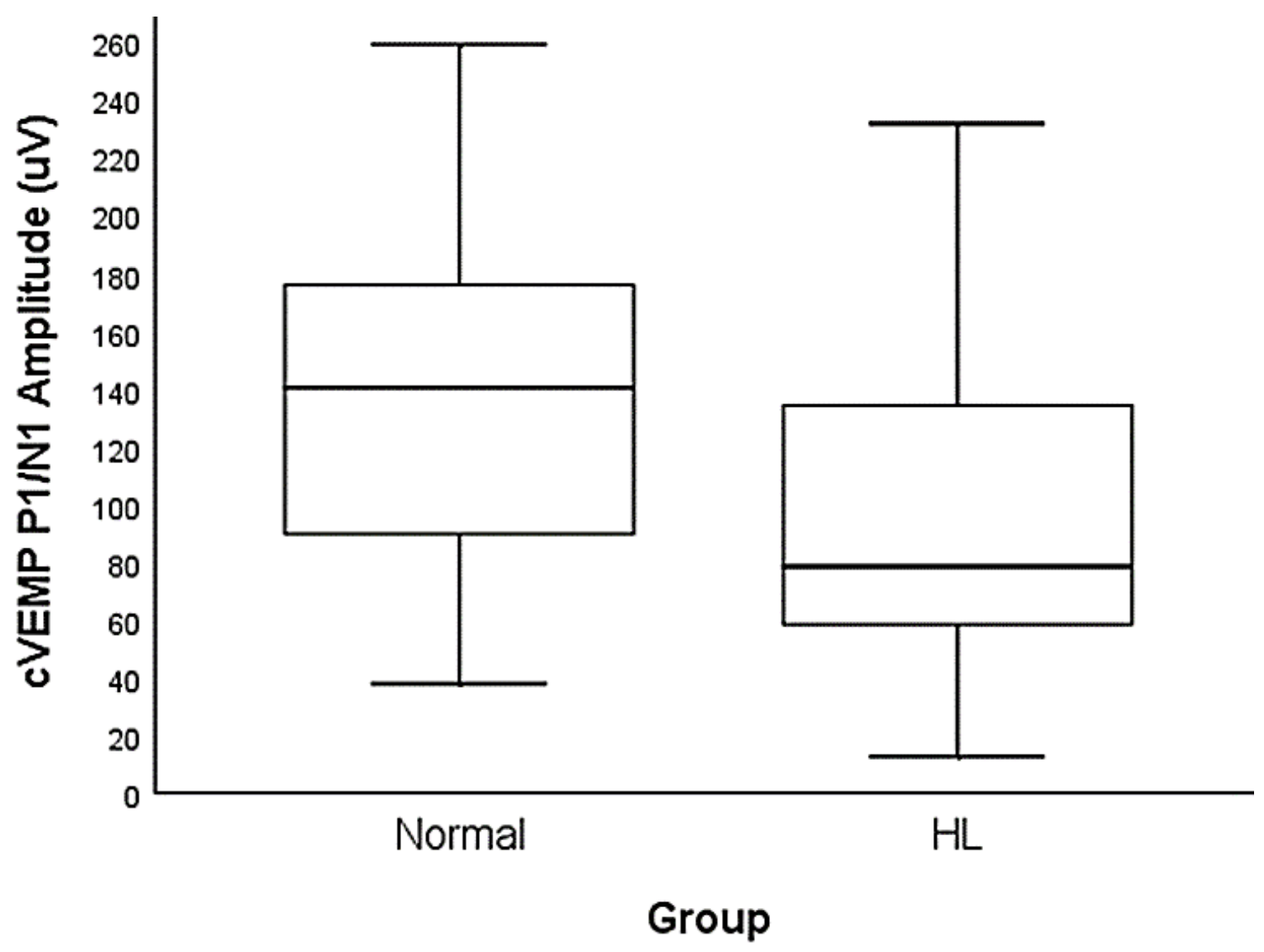

Figure 5.1: Mean cVEMP Amplitude for Group.

\pm 2 Standard Deviations shown 


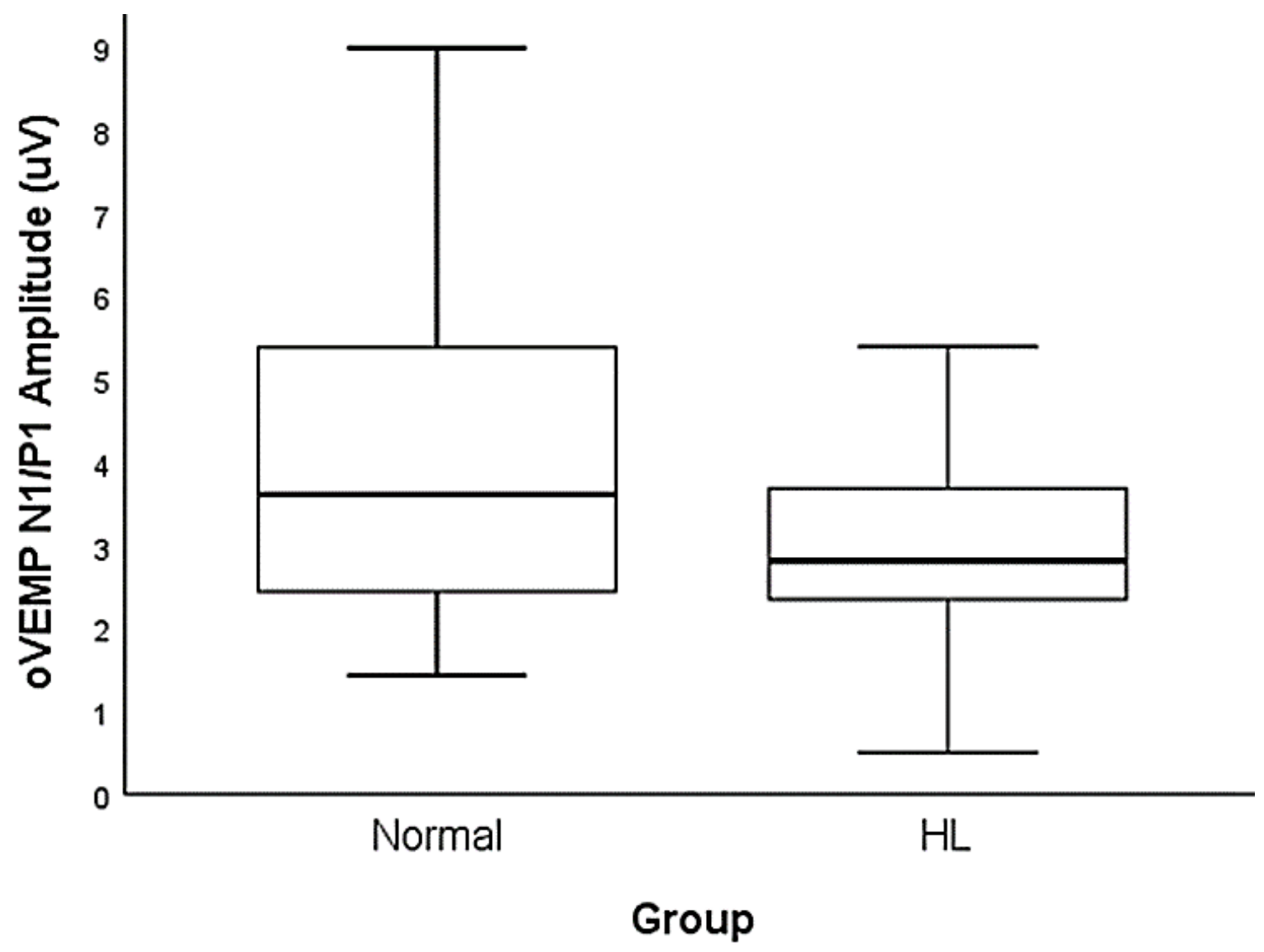

Figure 5.2: Mean cVEMP Amplitude of Normal and ARHL Groups.

\pm 2 Standard Deviations shown 
Table 5.2: Independent Samples T-Test for Group Differences in Mean for Postural Stability Measures.

\begin{tabular}{lccccccc}
\hline $\begin{array}{l}\text { Postural } \\
\text { Stability } \\
\text { Measures }\end{array}$ & $\mathbf{t}$ & df & $\begin{array}{c}\text { P- } \\
\text { Value }\end{array}$ & $\begin{array}{c}\text { Mean } \\
\text { Diff. }\end{array}$ & $\begin{array}{c}\text { Std. } \\
\text { Error } \\
\text { Diff. }\end{array}$ & $\begin{array}{c}\text { 95\% Confidence } \\
\text { Interval of the } \\
\text { Difference }\end{array}$ \\
\hline $\begin{array}{l}\text { Double } \\
\text { Leg }\end{array}$ & -1.060 & 14.084 & 0.270 & -0.176 & 0.168 & -0.540 & 0.183 \\
Lower & Upper \\
$\begin{array}{l}\text { Tandem } \\
\text { Right }\end{array}$ & -0.863 & 26 & 0.396 & -0.629 & 0.727 & -2.126 & 0.869 \\
$\begin{array}{l}\text { Tandem } \\
\text { Left }\end{array}$ & 2.006 & 16.366 & 0.062 & 0.637 & 0.316 & -0.035 & 1.309 \\
FRT & & & & & & & \\
\hline
\end{tabular}

Table 5.3: Pearson Correlation for Mid-Frequency PTA and VEMP Measures.

\begin{tabular}{lccc}
\hline VEMP Measures & R-Squared & P-Value & N \\
\hline cVEMP P1 Latency & -0.122 & 0.176 & 60 \\
cVEMP N1 Latency & -0.138 & 0.146 & 60 \\
cVEMP P2 Latency & 0.008 & 0.475 & 60 \\
cVEMP P1/N1 Amplitude & -0.359 & 0.002 & 60 \\
oVEMP N1 Latency & 0.154 & 0.123 & 59 \\
oVEMP P1 Latency & 0.292 & 0.012 & 59 \\
oVEMP N1/P1 Amplitude & -0.234 & 0.036 & 60 \\
\hline
\end{tabular}


Table 5.4: Benjamin-Hochberg Correction for Multiple Comparisons for VEMP Measures from Table 5.3.

\begin{tabular}{lccccc}
\hline $\begin{array}{l}\text { VEMP } \\
\text { Measures }\end{array}$ & P-Value & Rank & B-H Equation (i/m)Q & B-H Value & $\begin{array}{c}\text { Significance } \\
\text { (Yes/No) }\end{array}$ \\
\hline cVEMP Amp & 0.008 & 1 & $1 / 7 \times .05$ & 0.007 & $\mathrm{Y}$ \\
oVEMP P1 & 0.012 & 2 & $2 / 7 \times .05$ & 0.014 & $\mathrm{Y}$ \\
oVEMP Amp & 0.036 & 3 & $3 / 7 \times .05$ & 0.021 & $\mathrm{~N}$ \\
oVEMP N1 & 0.123 & 4 & $4 / 7 \times .05$ & 0.029 & $\mathrm{~N}$ \\
cVEMP N1 & 0.146 & 5 & $5 / 7 \times .05$ & 0.036 & $\mathrm{~N}$ \\
cVEMP P1 & 0.176 & 6 & $6 / 7 \times .05$ & 0.043 & $\mathrm{~N}$ \\
cVEMP P2 & 0.475 & 7 & $7 / 7 \times .05$ & 0.050 & $\mathrm{~N}$ \\
\hline
\end{tabular}

Table 5.5: Pearson Correlation for ARHL Group for Low-Frequency PTA, High-Frequency PTA and Hearing Loss Slope and VEMP Amplitudes.

\begin{tabular}{llcc}
\hline Audiometric Value & Statistical Values & cVEMP Amplitude & oVEMP Amplitude \\
\hline Low Hz PTA & R-squared & -.635 & 0.037 \\
& P-value & 0.000 & 0.428 \\
High Hz PTA & N & 27 & 27 \\
& R-squared & 0.292 & .397 \\
& P-value & 0.070 & 0.020 \\
HL Slope & N & 27 & 27 \\
& R-squared & -.628 & -.378 \\
& P-value & 0.000 & 0.026 \\
\hline
\end{tabular}

Table 5.6: Benjamin-Hochberg Correction for Multiple Comparisons for Low-Frequency PTA from Table 5.5.

\begin{tabular}{lccccc}
\hline $\begin{array}{l}\text { VEMP } \\
\text { Measures }\end{array}$ & P-Value & Rank & B-H Equation (i/m)Q & B-H Value & $\begin{array}{c}\text { Significance } \\
\text { (Yes/No) }\end{array}$ \\
\hline cVEMP AMP & 0.000 & 1 & $1 / 2 \times .05$ & 0.025 & $\mathrm{Y}$ \\
oVEMP AMP & 0.428 & 2 & $2 / 2 \times .05$ & 0.050 & $\mathrm{~N}$ \\
\hline
\end{tabular}




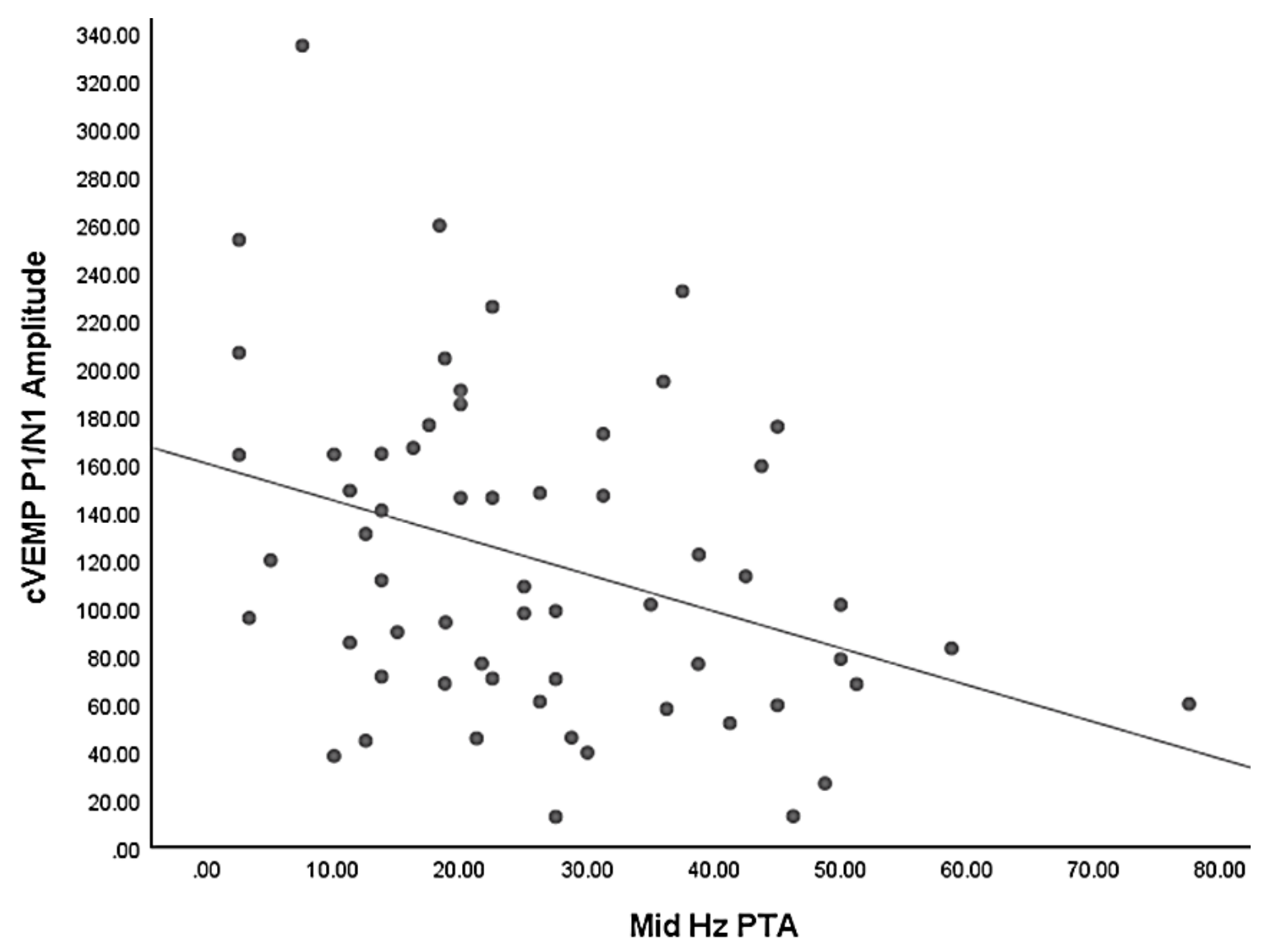

Figure 5.3: Correlation of Mid-Frequency PTA to cVEMP P1/N1 Amplitude.

Showing the linear regression correlation line $\mathrm{R}=0.129$

Table 5.7: Benjamin-Hochberg Correction for Multiple Comparisons for High-Frequency PTA from Table 5.5.

\begin{tabular}{lccccc}
\hline $\begin{array}{l}\text { VEMP } \\
\text { Measures }\end{array}$ & P-Value & Rank & B-H Equation (i/m)Q & B-H Value & $\begin{array}{c}\text { Significance } \\
\text { (Yes/No) }\end{array}$ \\
\hline oVEMP AMP & 0.020 & 1 & $1 / 2 \times .05$ & 0.025 & $\mathrm{Y}$ \\
cVEMP AMP & 0.070 & 2 & $2 / 2 \times .05$ & 0.050 & $\mathrm{~N}$ \\
\hline
\end{tabular}




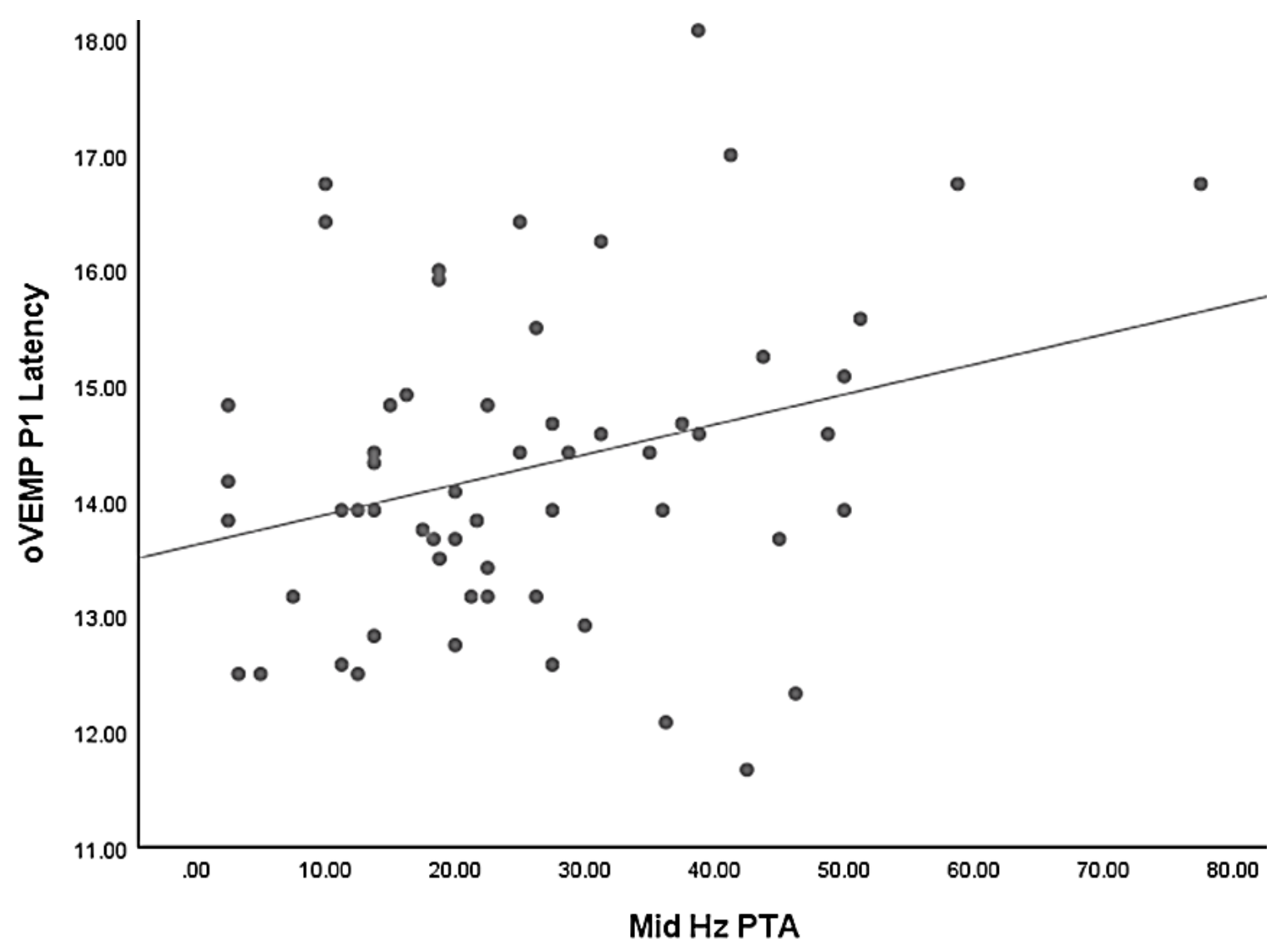

Figure 5.4: Correlation of Mid-Frequency PTA to oVEMP P1 Latency.

Showing the linear regression correlation line $\mathrm{R}=0.085$

Table 5.8: Benjamin-Hochberg Correction for Multiple Comparisons for Hearing Loss Slope from Table 5.5.

\begin{tabular}{lccccc}
\hline $\begin{array}{l}\text { VEMP } \\
\text { Measures }\end{array}$ & P-Value & Rank & B-H Equation (i/m)Q & B-H Value & $\begin{array}{c}\text { Significance } \\
\text { (Yes/No) }\end{array}$ \\
\hline cVEMP AMP & 0.000 & 1 & $1 / 2 \times .05$ & 0.025 & Y \\
oVEMP AMP & 0.026 & 2 & $2 / 2 x .05$ & 0.050 & Y \\
\hline
\end{tabular}




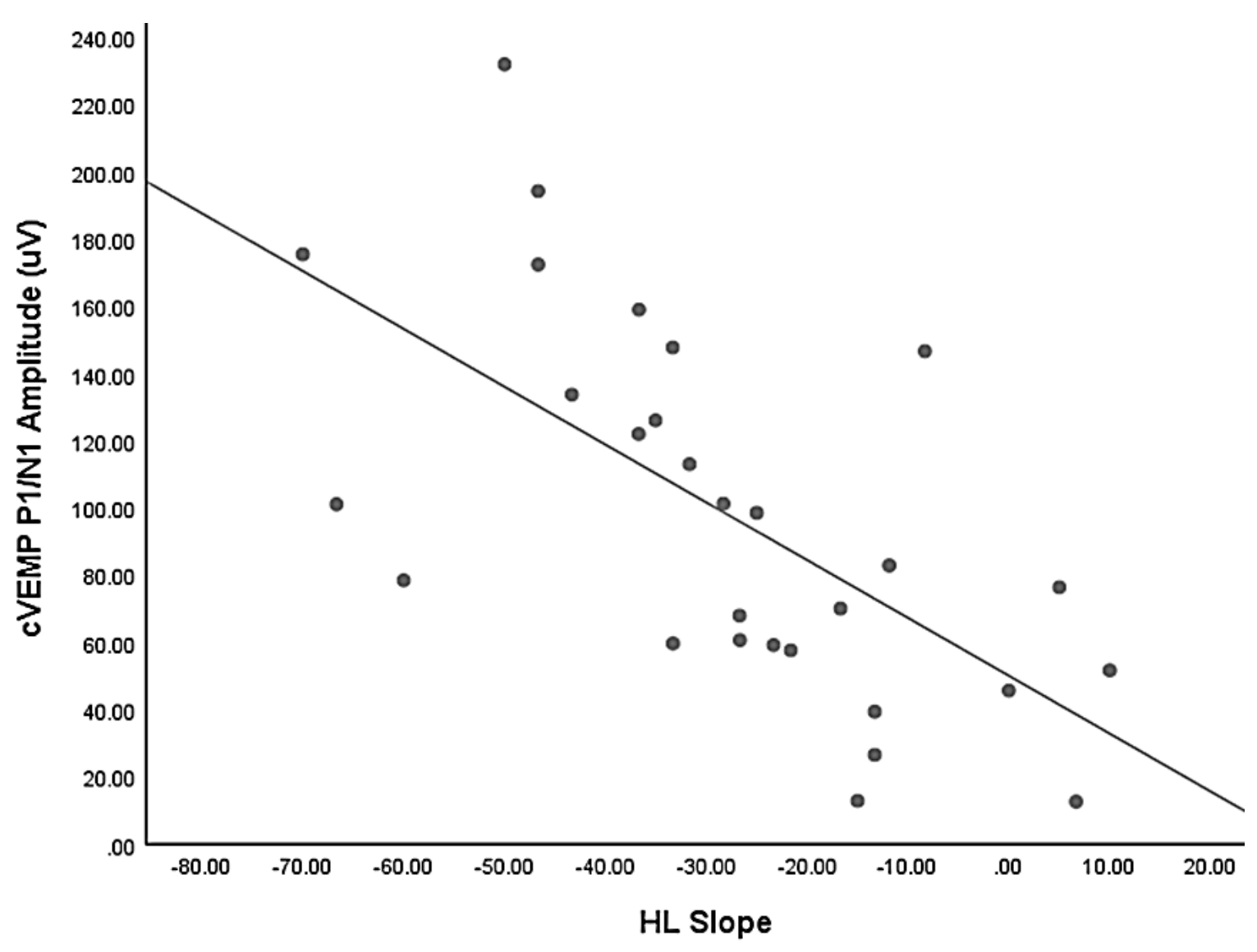

Figure 5.5: Correlation of cVEMP P1/N1 Amplitude and HL Slope for the ARHL Group Only.

Showing the linear regression correlation line $\mathrm{R}=0.406$ 


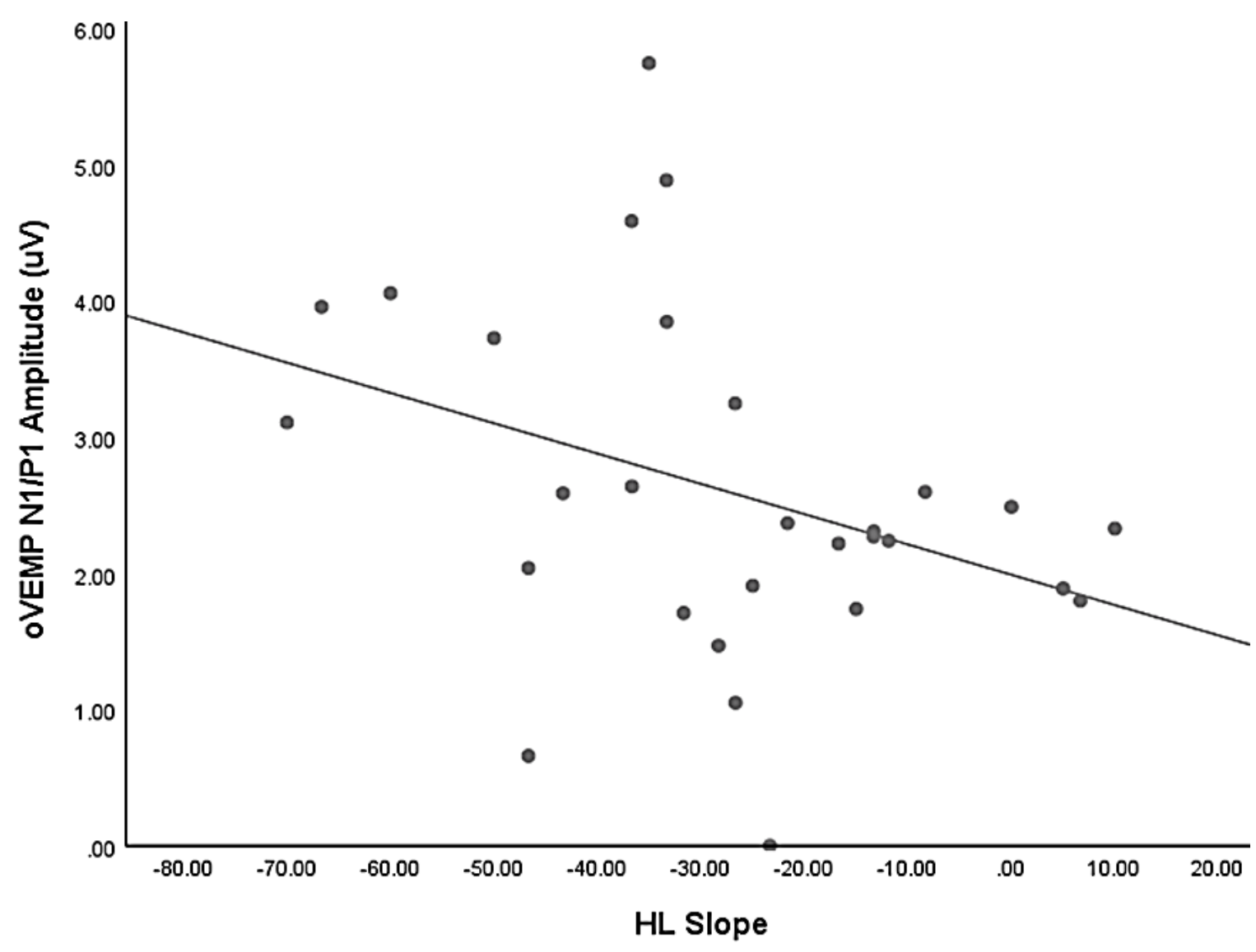

Figure 5.6: Correlation of oVEMP N1/P1 Amplitude and HL Slope for the ARHL Group Only.

Showing the linear regression correlation line $\mathrm{R}=0.132$ 


\section{Chapter 6}

\section{DISCUSSION AND CONCLUSIONS}

\subsection{Discussion}

The primary purpose of this study was to evaluate if there is a significant difference in saccule/utricle function as measured by cVEMP and oVEMP testing and postural control measured by KD Balance and FRT scores between older adults with normal hearing and older adults with ARHL. The results of this study indicated a significant difference in cVEMP P1/N1 amplitude and oVEMP N1/P1 amplitude between adults 50-70-years of age with normal hearing and adults in the same age range with ARHL. The adults in the ARHL group exhibited significantly decreased cVEMP P1/N1 and oVEMP n1/P1 amplitudes than the normal hearing group. There were no significant group differences for cVEMP or oVEMP latencies, KD Balance scores or FRT scores.

These results are consistent with previous findings from Kurtaran et al., 2016 who also reported significantly reduced cVEMP P1/N1 amplitudes in participants with ARHL. In contrast, the Kurtaran study also reported significant differences for cVEMP P1 and N1 latency values as well with delayed latencies in the ARHL group. Differences in these results and the Kurtaran et al., 2016 study could be related to differences in qualification of participants for group as they did not report the PTA frequencies or criteria for groups. Additional consideration for the differences in latency findings could be given to the age range and degree of hearing loss in the ARHL group. The Kurtaran et al., 2016 study had a wider age range (55-82-years of age) and a higher degree of hearing loss in their ARHL group which could account for increased progression of the underlying shared pathophys-

iology of age-related changes in hearing and vestibular function as suggested in both studies.

The results of this study indicated significantly decreased oVEMP P1/N1 amplitude for the ARHL participants. No studies at the time of this publication are known to have evaluated group differences for oVEMP values eliminating the possibility of comparisons to previous literature. However, these results are consistent with at least some of the previous histopathological studies. Johnsson, 1971 and Park et al., 2001 both described significant degeneration of saccular otoconia and nerve networks with effect to the utricle present to a lesser degree. Schuknecht, Icarashi, and Gacek, 1965, however, described 
pathological changes resulting in cochlea-saccular degeneration as a result of aging, but noted that the utricle and semicircular canals (SCCs) appeared to be intact. Schuknecht, Icarashi, and Gacek, 1965 did note, however, that challenges in evaluation of utricle due to damage during the investigation of the saccule. Our results and the histopathological data suggest a pattern of degeneration with both the saccule and utricle affected with the former exhibiting the largest effects. These results also suggest that VEMPs are a sensitive measure of the note histopathological degradation.

This pattern of degradation is also consistent with previous literature from the investigations of concomitant auditory and vestibular effects in the NIHL which suggested a model of degeneration based on the spread (and decrease) of energy from the saccule, utricle, and SCCs (Juntunen et al., 1987; Kilburn, Warshaw, and Hanscom, 1992; Golz et al., 2001; Wang and Young, 2007; Akdogan et al., 2009; Kumar, Vivarthini, and Bhat, 2010; Tseng and Young, 2012). Comparisons can also be made to the pattern of hearing loss in ARHL with the high-frequency portion of the cochlea showing the most degeneration due to its proximity to the oval window and the spread of energy. These results also are consistent with the findings from evaluations of concomitant auditory and vestibular dysfunction in SNHL. Sazgar et al., 2006, Hong et al., 2008, and Xu et al., 2016 all reported significant decreases in vestibular function as measured by VEMPs. Xu et al., 2016 also reported significant effects for both cVEMP and oVEMP, with more significant effects for saccular function.

Correlation results from this study also described these effects with a significant correlation of mid-frequency PTA and cVEMP P1/N1 amplitude as well as oVEMP P1 latency. The correlation results are also consistent with Zuniga et al., 2012 who also described significant correlations with low-, mid-, and high-frequency PTAs (with high-frequency PTA have the strongest relationship) with decreased cVEMP amplitude. Interestingly, Zuniga et al., 2012 also indicated significant correlations with both low- and high-frequency PTAs in addition to mid-frequency PTAs. Further analysis of our data using low-frequency PTA $(250,500,1000 \mathrm{~Hz})$ and high-frequency PTA $(4000,6000,8000 \mathrm{~Hz})$ also indicated several significant correlations. These results indicated a significant correlation of the low-frequency PTA with decreased cVEMP P1/N1 amplitude and high-frequency PTA with oVEMP amplitude suggested at least some possible frequency affects. Based on the findings from our study as well as Zuniga et al., 2012 post-hoc analysis of the relationship of degree of hearing loss and frequency of hearing loss was completed. HLS was calculated for each participant as a manner of capturing the frequency dynamic of the participants with ARHL. Correlational analysis of HLS indicated a significant correlation of decreased slope with decreased cVEMP P1/N1 and oVEMP N1/P1 amplitudes with decreased HLS.

Interestingly, these results of this study did not indicate any significant group differences or correlations with ARHL or PTAs to KD Balance or FRT scores. Considering the strong literature supporting decreased postural stability with decreased hearing from the systematic review of Agmon, Lavie, and Doumas, 2017 it could be suggested that the KD Balance and FRT measures failed to capture this dysfunction. It has been postulated 
that the vestibular dysfunction related to hearing loss is subclinical in nature making it difficult to measure with testing protocols normed for abnormal function. Viljanen et al., 2009 indicated that increased hearing loss (mean thresholds level for $500-4000 \mathrm{~Hz}$ in $\mathrm{dB}$ ) correlated with a higher risk for falls and decreased postural control. Lin and Ferrucci, 2012 also evaluated hearing and falls in the elderly population and reported a significant association between hearing loss and the odds of reported falls with every $10-\mathrm{dB}$ increase in hearing loss increasing the odds a fall. The results of this study suggest a connection between the degradation of hearing and vestibular dysfunction but did not indicate the same relationship for the postural control measured evaluated.

Limitations of study include the possibility of a population bias as the participants were recruited from a regional area and results may not be representative of the larger population. Additionally, the possibility of a Type I error is always present and could be reduced with a larger sample size. The results of this study suggested reduced postural control concomitant with decreased hearing, however the actual risk of falls was not calculated in this study. Other considerations in this study include the group cut-offs for placing participants in either the normal hearing or ARHL groups. We decided to use the WHO definition of hearing loss which is a PTA of greater than $25 \mathrm{~dB}$, however an argument could be made for a lower cut-off PTA $(500,1000,2000,4000 \mathrm{~Hz}) \mathrm{dB}$ level (i.e. 15 or 20dB) or different PTA measures representing different frequencies could have an effect on these results. Specifically, the results of this study suggested that low-frequency hearing loss could have a stronger association with vestibular dysfunction than either mid- or high-frequency hearing in this study group, however the possibility of subclinical conductive pathology in the middle ear system resulting in stiffness and reduced amplitude of stimulation during both audiometric and AC VEMP stimulation was not measured in this study.

\subsection{Conclusions}

Overall, using the histopathological framework set forth by Schuknecht, Icarashi, and Gacek, 1965 and Johnsson, 1971, the results of this study describe auditory and concomitant vestibular degeneration involving the saccule and to a lesser degree the utricle. In addition, the results of this study suggest the possibility of a frequency affect with lowfrequency hearing loss or HLS. This suggests the possibility that two specific types of presbycusis as described by Schuknecht, 1964; Schuknecht and Gacek, 1993 may be more associated with vestibular degradation at least for the age-group investigated. Metabolic (strial) presbycusis is theorized to result from metabolic and vascular changes causing a reduction in cochlear electrical potentials and energy production and is associated with a flat SNHL with very good speech discrimination. Additionally, mechanical (cochlear conductive) presbycusis results in alterations of cochlear mechanics due to changes in mass/stiffness properties and/or spiral ligament atrophy associated with gradual sloping hearing loss with slow progression. It is possible that people with these types of presbycusis could be at increased risk for vestibular dysfunction, although further study will be 
needed to confirm that supposition. It could also be postulated that based on these results and other literature that that hair cell and otoconia loss are not likely the only significant factor in decreased vestibular function. In fact, the description from Park et al., 2001 of vestibular neural ganglion loss could also explain the possible degradation of both saccule and utricle function by a shared underlying pathophysiology.

These results also lend credence to the supposition from Johnsson, 1971 that "mechanical wear" of the vestibular structures over time make the saccule more susceptible to degradation due to its gravitational orientation. Additional theories coming from the studies involving NIHL and SNHL also established a sequence of degenerative changes in NIHL as measured with diagnostic testing with audiometry, cVEMP, oVEMP, and calorics which is also consistent with the results of this study. Based on these results and limitations, further analysis of the relationship between ARHL, vestibular dysfunction, and postural control should include different PTA criteria (grouping and frequencies) for ARHL, with specific interest in low-frequency hearing thresholds and/or HLS. In addition, followup studies should include a measure of falls risk and/or monitor falls in the study population longitudinally to evaluate if these noted concomitant decreases in hearing and vestibular function result in increased falls. The long-term goal of this line of this study is to evaluate the use of auditory function as a predictor of increased risk of falls and a possible criterion for subsequent balance function testing and intervention as needed as a means to reduce the risk and occurrence of falls in people with ARHL. 


\section{List of References}

Age-Related Hearing Loss (Aug. 18, 2015). NIDCD. Library Catalog: www.nidcd.nih.gov. URL: https : / / www . nidcd.nih . gov/health/age-related-hearing-loss (visited on 03/25/2020).

Agmon, Maayan, Limor Lavie, and Michail Doumas (June 2017). "The Association between Hearing Loss, Postural Control, and Mobility in Older Adults: A Systematic Review". In: Journal of the American Academy of Audiology 28.6, pp. 575-588. ISSN: 2157-3107. DOI: $10.3766 /$ jaaa. 16044.

Agrawal, Yuri, Elizabeth A. Platz, and John K. Niparko (July 28, 2008). "Prevalence of Hearing Loss and Differences by Demographic Characteristics Among US Adults: Data From the National Health and Nutrition Examination Survey, 1999-2004". In: Archives of Internal Medicine 168.14. Publisher: American Medical Association, pp. 1522-1530. ISSN: 0003-9926. DOI: 10 . 1001 / archinte . 168 . 14 . 1522. URL: https : / / jamanetwork . com/ journals/jamainternalmedicine/fullarticle/414406 (visited on 03/30/2020).

Akdogan, Ozgur et al. (Feb. 1, 2009). "Continuous or intermittent noise exposure, does it cause vestibular damage?: An experimental study". In: Auris Nasus Larynx 36.1, pp. 2-6. ISSN: 0385-8146. DOI: $10.1016 / j$. anl . 2008.03 .003. URL: http: //www . sciencedirect.

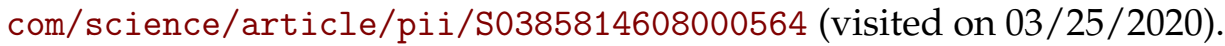

Bai, U. et al. (July 1997). "Mitochondrial DNA deletions associated with aging and possibly presbycusis: a human archival temporal bone study". In: The American Journal of Otology 18.4, pp. 449-453. ISSN: 0192-9763.

Basta, Dietmar, Ingo Todt, and Arne Ernst (2007). "Characterization of age-related changes in vestibular evoked myogenic potentials". In: Journal of Vestibular Research: Equilibrium $\mathcal{E}$ Orientation 17.2, pp. 93-98. ISSN: 0957-4271.

Bergen, Gwen, Mark R. Stevens, and Elizabeth R. Burns (Sept. 23, 2016). "Falls and Fall Injuries Among Adults Aged $\geq 65$ Years - United States, 2014". In: MMWR. Morbidity and mortality weekly report 65.37, pp. 993-998. ISSN: 1545-861X. DOI: 10 . 15585 / mmwr . mm6537a2.

Bischoff, Heike A. et al. (May 2003). "Identifying a cut-off point for normal mobility: a comparison of the timed 'up and go' test in community-dwelling and institutionalised elderly women". In: Age and Ageing 32.3, pp. 315-320. ISSN: 0002-0729. DOI: 10 . 1093 / ageing/32.3.315.

Brant, L. J. and J. L. Fozard (Aug. 1990). "Age changes in pure-tone hearing thresholds in a longitudinal study of normal human aging". In: The Journal of the Acoustical Society of America 88.2, pp. 813-820. ISSN: 0001-4966. DOI: 10.1121/1.399731. 
Colebatch, J.G. and G.M. Halmagyi (Aug. 1992). "Vestibular evoked potentials in human neck muscles before and after unilateral vestibular deafferentation". In: Neurology 42.8, pp. 1635-1636. ISSN: 0028-3878. DOI: 10.1212/wnl.42.8.1635.

Colebatch, J.G., G.M. Halmagyi, and N.F. Skuse (Feb. 1994). "Myogenic potentials generated by a click-evoked vestibulocollic reflex." In: Journal of Neurology, Neurosurgery, and Psychiatry 57.2, pp. 190-197. ISSN: 0022-3050. URL: https : / www . ncbi .nlm . nih . gov / pmc/articles/PMC1072448/ (visited on 03/25/2020).

Crowe, S., R. Guild, and M. Polvogt (Oct. 1934). "Observations on the Pathology of HighTone Deafness". In: The Journal of Nervous and Mental Disease 80.4, p. 480. ISSN: 0022-3018. URL: https : / / journals. lww . com/ jonmd/Citation/1934/10000/Observations_on_ the_Pathology_of_High_Tone.23.aspx (visited on 03/25/2020).

Cruickshanks, K. J. et al. (Nov. 1, 1998). "Prevalence of hearing loss in older adults in Beaver Dam, Wisconsin. The Epidemiology of Hearing Loss Study". In: American Journal of Epidemiology 148.9, pp. 879-886. ISSN: 0002-9262. DOI: 10.1093/oxfordjournals . aje. a009713.

Curthoys, I.S., V. Vulovic, and L. Manzari (Feb. 2012). “Ocular vestibular-evoked myogenic potential (oVEMP) to test utricular function: neural and oculomotor evidence". In: Acta Otorhinolaryngologica Italica 32.1, pp. 41-45. ISSN: 0392-100X. URL: https : / /www . ncbi . nlm.nih.gov/pmc/articles/PMC3324959/ (visited on 03/25/2020).

Duncan, P. W., S. Studenski, et al. (May 1992). "Functional reach: predictive validity in a sample of elderly male veterans". In: Journal of Gerontology 47.3, pp. M93-98. ISSN: 00221422. DOI: 10.1093/geronj/47.3.m93.

Duncan, P. W., D. K. Weiner, et al. (Nov. 1990). "Functional reach: a new clinical measure of balance". In: Journal of Gerontology 45.6, pp. M192-197. ISSN: 0022-1422. DOI: 10.1093/ geronj/45.6.m192.

Fetoni, Anna R. et al. (June 2011). "Pathogenesis of presbycusis in animal models: a review". In: Experimental Gerontology 46.6, pp. 413-425. ISSN: 1873-6815. DOI: $10.1016 / \mathrm{j}$. exger.2010.12.003.

Gimsing, S. (Mar. 2010). "Vestibular schwannoma: when to look for it?" In: The Journal of Laryngology $\mathcal{E}$ Otology 124.3. Publisher: Cambridge University Press, pp. 258-264. ISSN: 1748-5460, 0022-2151. DOI: 10 . 1017 / S0022215109991423. URL: https : / / www . cambridge . org / core / journals / journal - of - laryngology - and - otology / article / vestibular-schwannoma-when-to-look-for-it/F25B9031DCF1571CD43E95670EA6F06A (visited on 03/25/2020).

Golz, A. et al. (June 2001). "The effects of noise on the vestibular system". In: American Journal of Otolaryngology 22.3, pp. 190-196. ISSN: 0196-0709. DOI: 10 . 1053/ajot . 2001. 23428.

Gopinath, Bamini et al. (Feb. 23, 2009). "Prevalence of age-related hearing loss in older adults: Blue Mountains Study". In: Archives of Internal Medicine 169.4, pp. 415-416. ISSN: 1538-3679. DOI: 10.1001/archinternmed.2008.597.

Guidelines for Manual Pure-Tone Threshold Audiometry (2020). American Speech-LanguageHearing Association. Library Catalog: www.asha.org Publisher: American 
Speech-Language-Hearing Association. URL: https : / www . asha . org/policy/GL200500014/ (visited on 03/25/2020).

Hageman, P. A., J. M. Leibowitz, and D. Blanke (Oct. 1995). “Age and gender effects on postural control measures". In: Archives of Physical Medicine and Rehabilitation 76.10, pp. 961965. ISSN: 0003-9993. DOI: 10.1016/s0003-9993(95)80075-1.

Helzner, Elizabeth P. et al. (Dec. 2005). "Race and sex differences in age-related hearing loss: the Health, Aging and Body Composition Study". In: Journal of the American Geriatrics Society 53.12, pp. 2119-2127. ISSN: 0002-8614. DOI: 10 .1111/ j . 1532-5415 . 2005. 00525.x.

Hong, Seok Min et al. (Oct. 1, 2008). "Saccular damage in patients with idiopathic sudden sensorineural hearing loss without vertigo". In: Otolaryngology - Head and Neck Surgery 139.4, pp. 541-545. ISSN: 0194-5998. DOI: 10.1016/j . otohns . 2008.07 .003. URL: http: / / www . sciencedirect . com/science / article / pii / S019459980801190X (visited on 03/25/2020).

Horak, F. B., C. L. Shupert, and A. Mirka (Dec. 1989). “Components of postural dyscontrol in the elderly: a review". In: Neurobiology of Aging 10.6, pp. 727-738. ISSN: 0197-4580. DOI: 10.1016/0197-4580 (89)90010-9.

Hornsby, Benjamin W. Y., Earl E. Johnson, and Erin Picou (2011). "Effects of degree and configuration of hearing loss on the contribution of high- and low-frequency speech information to bilateral speech understanding". In: Ear and hearing 32.5, pp. 543-555. ISSN: 0196-0202. DOI: 10 . 1097 / AUD . 0b013e31820e5028. URL: https : //www . ncbi .nlm . nih.gov/pmc/articles/PMC3113662/ (visited on 03/25/2020).

Janky, Kristen L. and Neil Shepard (Sept. 2009). "Vestibular Evoked Myogenic Potential (VEMP) Testing: Normative Threshold Response Curves and Effects of Age". In: Journal of the American Academy of Audiology 20.8, pp. 514-522. ISSN: 1050-0545. URL: https: //www.ncbi.nlm.nih.gov/pmc/articles/PMC2749261/ (visited on 03/25/2020).

Johnsson, L. G. (Oct. 1971). “Degenerative changes and anomalies of the vestibular system in man". In: The Laryngoscope 81.10, pp. 1682-1694. ISSN: 0023-852X. DOI: $10.1288 /$ 00005537-197110000-00016.

Juntunen, Juhani et al. (Aug. 1, 1987). "POSTURAL BODY SWAY AND EXPOSURE TO HIGH-ENERGY IMPULSE NOISE". In: The Lancet. Originally published as Volume 2, Issue 8553 330.8553, pp. 261-264. ISSN: 0140-6736. DOI: 10.1016/S0140-6736 (87) 908403. URL: http: //www . sciencedirect . com/science/article/pii/S0140673687908403 (visited on 03/25/2020).

Kear, Breelan M., Thomas P. Guck, and Amy L. McGaha (Jan. 2017). “Timed Up and Go (TUG) Test: Normative Reference Values for Ages 20 to 59 Years and Relationships With Physical and Mental Health Risk Factors". In: Journal of Primary Care E Community Health 8.1, pp. 9-13. ISSN: 2150-1327. DOI: 10.1177/2150131916659282.

Kilburn, K H, R H Warshaw, and B Hanscom (Feb. 1992). "Are hearing loss and balance dysfunction linked in construction iron workers?" In: British Journal of Industrial Medicine 49.2, pp. 138-141. ISSN: 0007-1072. URL: https : //www.ncbi.nlm.nih.gov/pmc/ articles/PMC1012079/ (visited on 03/25/2020). 
Kumar, Kaushlendra, Christina Jean Vivarthini, and Jayashree S. Bhat (Sept. 2010). "Vestibular evoked myogenic potential in noise-induced hearing loss". In: Noise $\mathcal{E}$ Health 12.48, pp. 191-194. ISSN: 1463-1741. DOI: 10.4103/1463-1741.64973.

Kurtaran, Hanifi et al. (Nov. 1, 2016). "The relationship between senile hearing loss and vestibular activity". In: Brazilian Journal of Otorhinolaryngology 82.6, pp. 650-653. ISSN: 1808-8694. DOI: 10 . 1016/ j . bjorl . 2015 . 11 . 016. URL: http : / /www . sciencedirect .

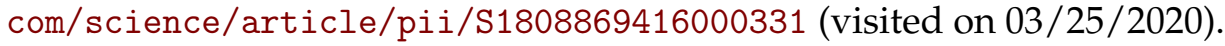

Li, Carol et al. (Nov. 2015). "Epidemiology of Vestibular Evoked Myogenic Potentials: Data from the Baltimore Longitudinal Study of Aging". In: Clinical neurophysiology : official journal of the International Federation of Clinical Neurophysiology 126.11, pp. 2207-2215. ISSN: 1388-2457. DOI: 10 . 1016/ j . clinph . 2015 . 01 . 008. URL: https : / / www . ncbi . nlm.nih.gov/pmc/articles/PMC4514573/ (visited on 03/25/2020).

Lin, Frank R. and Luigi Ferrucci (Feb. 27, 2012). "Hearing Loss and Falls Among Older Adults in the United States". In: Archives of internal medicine 172.4, pp. 369-371. ISSN: 0003-9926. DOI: 10.1001/archinternmed. 2011.728. URL: https://www .ncbi.nlm.nih . gov/pmc/articles/PMC3518403/ (visited on 03/25/2020).

Lin, Frank R., Kristine Yaffe, et al. (Feb. 25, 2013). "Hearing Loss and Cognitive Decline Among Older Adults". In: JAMA internal medicine 173.4. ISSN: 2168-6106. DOI: 10.1001/ jamainternmed . 2013 . 1868. URL: https : / / www . ncbi .nlm . nih.gov/pmc/articles / PMC3869227/ (visited on 03/25/2020).

Maki, B. E., P. J. Holliday, and G. R. Fernie (Jan. 1990). “Aging and postural control. A comparison of spontaneous- and induced-sway balance tests". In: Journal of the American Geriatrics Society 38.1, pp. 1-9. ISSN: 0002-8614. DOI: 10 . 1111 / j . $1532-5415$. 1990 . tb01588.x.

Mann, G. C. et al. (Oct. 1996). "Functional reach and single leg stance in patients with peripheral vestibular disorders". In: Journal of Vestibular Research: Equilibrium E Orientation 6.5, pp. 343-353. ISSN: 0957-4271.

McGill, T. J. and H. F. Schuknecht (Sept. 1976). "Human cochlear changes in noise induced hearing loss". In: The Laryngoscope 86.9, pp. 1293-1302. ISSN: 0023-852X. DOI: 10 . 1288 / 00005537-197609000-00001.

Mener, David J. et al. (Sept. 2013). "Hearing Loss and Depression in Older Adults". In: Journal of the American Geriatrics Society 61.9, pp. 1627-1629. ISSN: 0002-8614. DOI: 10 . 1111/jgs . 12429. URL: https : //www .ncbi .nlm.nih.gov/pmc/articles/PMC3773611/ (visited on 03/25/2020).

Nguyen, Kimanh D., Miriam S. Welgampola, and John P. Carey (July 2010). "Test-Retest Reliability and Age-related Characteristics of the Ocular and Cervical Vestibular Evoked Myogenic Potential Tests". In: Otology \& neurotology : official publication of the American Otological Society, American Neurotology Society [and] European Academy of Otology and Neurotology 31.5, pp. 793-802. ISSN: 1531-7129. DOI: 10 . 1097 / MAO . 0b013e3181e3d60e. URL: https : / / www . ncbi . nlm . nih . gov / pmc / articles / PMC2913294/ (visited on $03 / 25 / 2020)$. 
Ohlemiller, Kevin K. (Oct. 2004). "Age-related hearing loss: the status of Schuknecht's typology". In: Current Opinion in Otolaryngology E Head and Neck Surgery 12.5, pp. 439-443. ISSN: 1068-9508. DOI: 10.1097/01.moo.0000134450.99615.22.

Park, J. J. et al. (Mar. 19, 2001). “Age-related change in the number of neurons in the human vestibular ganglion". In: The Journal of Comparative Neurology 431.4, pp. 437-443. ISSN: 0021-9967. DOI: 10.1002/1096-9861 (20010319) 431:4<437: : aid-cne1081>3. 0 . co;2-p.

Report of the Informal Working Group on Prevention of Deafness and Hearing Impairment Programme Planning, Geneva, 18-21 June 1991 (2020). World Health Organization. URL: https : //apps.who.int/iris/handle/10665/58839 (visited on 03/25/2020).

Røgind, H. et al. (May 2003). "Postural sway in normal subjects aged 20-70 years". In: Clinical Physiology and Functional Imaging 23.3, pp. 171-176. ISSN: 1475-0961. DOI: 10 . 1046/j.1475-097x.2003.00492.x.

Saxen (1937). "Pathology and clinic in old age". In: Acta Oto-Laryngologica Supplemental 23.

Sazgar, Amir et al. (Aug. 1, 2006). "Saccular damage in patients with high-frequency sensorineural hearing loss". In: European archives of oto-rhino-laryngology : official journal of the European Federation of Oto-Rhino-Laryngological Societies (EUFOS) : affiliated with the German Society for Oto-Rhino-Laryngology - Head and Neck Surgery 263, pp. 608-13. DOI: 10.1007/s00405-006-0038-6.

Schuknecht (Oct. 1964). "FURTHER OBSERVATIONS ON THE PATHOLOGY OF PRESBYCUSIS". In: Archives of Otolaryngology (Chicago, Ill.: 1960) 80, pp. 369-382. ISSN: 0003-9977. DOI: 10.1001 /archotol.1964.00750040381003.

Schuknecht and Gacek (Jan. 1993). "Cochlear pathology in presbycusis". In: The Annals of Otology, Rhinology, and Laryngology 102.1, pp. 1-16. ISSN: 0003-4894. DOI: 10 . 1177 / 00034894931020 S101.

Schuknecht, Icarashi, and Gacek (Jan. 1, 1965). "The Pathological Types of Cochleo-Saccular Degeneration". In: Acta Oto-Laryngologica 59.2. Publisher: Taylor \& Francis, pp. 154-170. ISSN: 0001-6489. DOI: 10 . 3109/00016486509124549. URL: https : //doi . org/10 . 3109/ 00016486509124549.

Someya, Shinichi and Tomas A. Prolla (2010). "Mitochondrial oxidative damage and apoptosis in age-related hearing loss". In: Mechanisms of ageing and development 131.0, pp. 480486. ISSN: 0047-6374. DOI: $10.1016 / \mathrm{j} \cdot \operatorname{mad} .2010 .04$.006. URL: https : / www . ncbi . nlm . nih.gov/pmc/articles/PMC4086639/ (visited on 03/25/2020).

Steffen, Teresa M., Timothy A. Hacker, and Louise Mollinger (Feb. 2002). "Age- and genderrelated test performance in community-dwelling elderly people: Six-Minute Walk Test, Berg Balance Scale, Timed Up \& Go Test, and gait speeds". In: Physical Therapy 82.2, pp. 128-137. ISSN: 0031-9023. DOI: $10.1093 / \mathrm{ptj} / 82.2 .128$.

Su, Hsuan-Chao et al. (Nov. 1, 2004). "Aging Effect on Vestibular Evoked Myogenic Potential". In: Otology \& neurotology : official publication of the American Otological Society, American Neurotology Society [and] European Academy of Otology and Neurotology 25, pp. 977-80. DOI: $10.1097 / 00129492-200411000-00019$. 
Thyssen, H. H. et al. (July 1982). "Normal ranges and reproducibility for the quantitative Romberg's test". In: Acta Neurologica Scandinavica 66.1, pp. 100-104. ISSN: 0001-6314. DOI: 10.1111/j.1600-0404.1982.tb03133.x.

Todd, Neil P. McAngus et al. (Feb. 2007). “Ocular vestibular evoked myogenic potentials (OVEMPs) produced by air- and bone-conducted sound". In: Clinical Neurophysiology: Official Journal of the International Federation of Clinical Neurophysiology 118.2, pp. 381-390. ISSN: 1388-2457. DOI: 10.1016/j.clinph.2006.09.025.

Tseng, Chia-Chen and Yi-Ho Young (Nov. 10, 2012). "Sequence of vestibular deficits in patients with noise-induced hearing loss". In: European archives of oto-rhino-laryngology : official journal of the European Federation of Oto-Rhino-Laryngological Societies (EUFOS) : affiliated with the German Society for Oto-Rhino-Laryngology - Head and Neck Surgery 270. DOI: $10.1007 / \mathrm{s} 00405-012-2270-6$.

Viljanen, Anne et al. (Feb. 2009). "Hearing as a predictor of falls and postural balance in older female twins". In: The Journals of Gerontology. Series A, Biological Sciences and Medical Sciences 64.2, pp. 312-317. ISSN: 1758-535X. DOI: 10.1093/gerona/gln015.

Wang, Yen-Pin and Yi-Ho Young (Oct. 1, 2007). "Vestibular-evoked myogenic potentials in chronic noise-induced hearing loss". In: Otolaryngology - Head and Neck Surgery 137.4, pp. 607-611. ISSN: 0194-5998. DOI: $10.1016 / \mathrm{j}$. otohns . 2007 . 05 .005. URL: http : / / www . sciencedirect. com / science/article/pii / S0194599807005116 (visited on 03/25/2020).

$\mathrm{Xu}$, Xin-Da et al. (Jan. 1, 2016). "The hidden dysfunction of otolithic organs in patients with profound sensorineural hearing loss". In: Hearing Research 331, pp. 41-46. ISSN: 0378-5955. DOI: 10 . 1016/j . heares . 2015 . 10 . 006. URL: http : / www . sciencedirect .

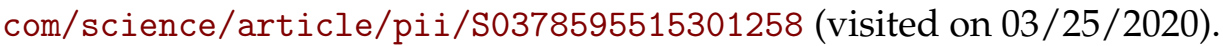

Zuniga, M. Geraldine et al. (Dec. 2012). "Association between Hearing Loss and Saccular Dysfunction in Older Individuals". In: Otology \& neurotology : official publication of the American Otological Society, American Neurotology Society [and] European Academy of Otology and Neurotology 33.9, pp. 1586-1592. ISSN: 1531-7129. DOI: \\10 . 1097 / MAO . Ob013e31826bedbc. URL: https://www.ncbi.nlm.nih.gov/pmc/articles/PMC3498596/ (visited on $03 / 25 / 2020$ ). 


\section{Vita}

Steven M. Doettl was born in Maryville, Tennessee in 1976. He attended the University of Tennessee, Knoxville, where he earned a Bachelor of Arts Degree in Audiology and Speech Pathology in 1999. He then attended the University of Tennessee, Knoxville and earned a Master of Arts Degree in Audiology in 2001 and a Doctor of Audiology Degree in 2004. Dr. Doettl joined the faculty at the University of Tennessee Health Science Center in 2010 and began work on the Doctor of Philosophy Degree in Speech and Hearing Science with a concentration in Hearing Science under the direction of Dr. Patrick Plyler in 2011 and graduated in 2020 . 